\title{
Multiple In-Cycle Transshipments with Positive Delivery Times
}

\author{
Nagihan Çömez \\ Faculty of Business Administration, Bilkent University, Bilkent, Ankara 06800, Turkey, comez@bilkent.edu.tr \\ Kathryn E. Stecke, Metin Çakanyıldırım \\ School of Management, University of Texas at Dallas, Richardson, Texas 75083, USA \\ kstecke@utdallas.edu, metin@utdallas.edu
}

\begin{abstract}
$\mathrm{W}$ e study a centralized inventory sharing system of two retailers that are replenished periodically. Between two replenishments, a unit can be transshipped to a stocked-out retailer from the other. It arrives a transshipment time later, during which the stocked-out retailer incurs backorder cost. Without transshipment, backorder cost is incurred until the next replenishment. Since the transshipment time is shorter than the time between two replenishments, transshipments can reduce the backorder cost at the stocked-out retailer and the holding costs at the other retailer. The system is directed by a centralized inventory manager, who minimizes the long-run average cost consisting of replenishment, holding, backorder, and transshipment costs. The transshipment policy is characterized by hold-back inventory levels, which are nonincreasing in the remaining time until the next replenishment. The transshipment policy differs from those in the literature because we allow for multiple transshipments between replenishments, positive transshipment times, and backorder costs. We also discuss the challenges associated with positive replenishment time and develop upper and lower bounds of average cost in this case. Bounds are numerically shown to have an average gap of $1.1 \%$. A heuristic solution is based on the upper bound and differs from the optimal cost by at most this gap.
\end{abstract}

Key words: multiple in-cycle transshipments; positive transshipment and replenishment times; hold-back levels; centralized system

History: Received: January 2009; Accepted: January 2011 by Jayashankar Swaminathan, after 3 revisions.

\section{Introduction}

To gain competitive advantage, a firm can reduce backorders by decreasing the delivery time of a product. A faster delivery results in quicker revenue collection and so improves cash position. Stock-outs at a retailer until the next replenishment cause backorders, which can be decreased either by increasing the inventory at the retailers or by sharing the inventory among cooperating retailers.

In a network of inventory sharing (pooling) retailers, each retailer serves a geographic territory. The customers of a stocked-out retailer often do not go to another retailer. Instead, a centralized inventory manager (IM) can consider transshipping a unit to the stocked-out retailer from another retailer. If the other retailer has plenty of inventory, the IM instructs the other retailer to transship a unit to the stocked-out retailer. This paper characterizes this notion of plenty, depending on the time until the next replenishment, delivery (transshipment and replenishment) times, demand, and several cost parameters.

Inventory is shared in many industries such as apparel, sporting goods, toys, furniture, and automotive
(Rudi et al. 2001) and in many companies such as Ingram Micro (a distributor of information technology products), Foot Locker (a shoe retailer), and FNAC (a French retailer for cultural products) (Ozdemir et al. 2006). Inventory sharing is also commonly practiced to increase repair parts availability either for internal operational use or for after-sales repair services. Grahovac and Chakravarty (2001) and Kukreja et al. (2001) consider transshipment models for repair parts of construction equipment, aircraft, and power-generating plants. According to Stalk et al. (1997), US car dealers satisfy up to $18 \%$ of their new vehicle customer demand by locating vehicles at another dealer. The common features of a transshipped product often include significant replenishment times from manufacturers along with a short selling season (Rudi et al. 2001) and fairly high product margins. Another feature is low and infrequent demand for the product because of its specific functionality and/or high variety within its product family. Orders for lowdemand products usually arrive in single units and monthly demand is often in two digits, for example, around 25 for a spare part (Zhao et al. 2005) or around 20 for an automobile type (Toyota.com 2007). These 
demands average less than one per day. For these, a low level of inventory can be kept at each retailer by relying on transshipments to handle stock-outs.

In practice, many transshipments can occur between two replenishments, called a replenishment cycle henceforth. Multiple transshipments per replenishment cycle expedite the fulfilment of demand occurring at a stocked-out retailer. Corsten and Gruen (2004) emphasize quick delivery by noting that customers are becoming less tolerant to stock-outs. However, inventory sharing literature, with several exceptions (e.g., Archibald et al. 1997, Grahovac and Chakravarty 2001, Zhao et al. 2008) does not consider multiple transshipments per cycle.

Transshipment cost, transshipment time, and opportunity cost of less inventory at the retailers should be considered to accurately evaluate the benefit of a transshipment policy. For example, when the transshipment time is positive, transshipping a unit cannot eliminate backordering the unit during transshipment but it reduces the delivery delay at the stocked-out retailer. Most studies assume that either the replenishments, or the transshipments, or both occur instantaneously (Archibald et al. 1997). This paper allows multiple transshipments per cycle, and positive transshipment and replenishment times. Holding and backorder costs are charged throughout a cycle to closely model real-life applications. Such application areas include automobile dealerships, construction, agriculture, and heavy equipment retailers as well as many spare parts supply chains. Spare parts are very important for original equipment manufacturers because they can contribute $40-80 \%$ of the profit despite having the smallest share of the total sales volume (Wu and Tew 2005).

We study a distribution system of two retailers coordinated by an IM. The retailers are replenished according to a given prespecified schedule. This schedule is constructed by considering the expected demands, the production schedule at the manufacturer, the distance between the manufacturer and retailers, replenishment lead time, and the fixed cost of replenishment. This is why the replenishment schedule is not altered to consider the retailer demand realizations.

A stocked-out retailer places a transshipment request to the other (requested) retailer upon receiving a customer demand. If the requested retailer is instructed by the IM to accept the request, then she sends a unit to the requesting retailer. Otherwise, the requesting retailer incurs a backorder cost until the end of the cycle. It takes each unit a constant transshipment time to arrive at the requesting retailer, during which a backorder cost is incurred.

This paper presents an optimal transshipment policy, which is both implementable in many real-life applications and robust against different demand realizations as well as some demand parameters. The optimal transshipment policy of a retailer is characterized by hold-back levels at that retailer who transships inventory only when her current inventory level is more than her hold-back level. While the replenishment quantities are usually decision variables in the literature, hold-back levels are often given by a rule. They are zero under complete pooling policies (e.g., Tagaras 1999). In a more general scenario, however, a retailer may want to keep some inventory for herself as safety stock by practicing partial pooling (rationing), which is characterized by positive holdback levels. Partial pooling may be more beneficial in the presence of backordered demands, positive transshipment costs, and transshipment times.

The IM computes basestock quantities for replenishments and hold-back levels to minimize the expected long-run average system cost consisting of replenishment, inventory holding, backorder, and transshipment costs. The IM passes basestock quantities and holdback levels to the retailers to coordinate the system for many cycles (over an infinite horizon). After specifying the replenishment and transshipment policies, the IM is no longer involved in the operational decision making. This is important in real life because it lets the IM focus on other more strategic issues.

The details of our model are mainly motivated by actual automotive transshipments among dealers of auto makers such as Ford Motor Company. As transshipment applications are observed in various industries, the application details and restrictions can vary among various industries and companies. We discuss some extensions to model transshipments under different circumstances in detail in section 6.

The rest of the paper is organized as follows. Section 2 reviews the literature and our contributions. The long-run average cost is developed in section 3. Then the optimality and properties of the hold-back levels are discussed. In section 4, analytical and numerical studies are reported to examine the sensitivity of the optimal policies to system parameters and to show the performance of our transshipment policy in terms of cost reductions. In section 5, a heuristic is developed to deal with positive replenishment time. Our modeling assumptions are critically reviewed and extensions of our model are discussed in section 6 . Section 7 concludes the paper. All proofs are contained in the supporting information appendix.

\section{Related Literature and Our Contributions}

The idea of inventory sharing originates from inventory centralization (Berman et al. 2011). The early studies on inventory sharing by Krishnan and Rao 
(1965) and Gross (1963) focus on centralized multilocation transshipment systems. Krishnan and Rao allow a transshipment after all demand realizations, which is called an emergency lateral transshipment. On the other hand, Gross redistributes the inventories among retailers before the demands are realized, which is called preventive transshipment. Das (1975) extends the model of Gross (1963) by allowing transshipment in the middle of each period for the redistribution of inventories. Some later studies followed these two streams: transshipments before or after demand realizations.

Periodic review models are common in the transshipment literature (Karmarkar 1987, Klein 1990), where at the beginning of each period, retailer inventories are replenished. After the demand realization at the end of a period, lateral transshipment is allowed between the stocked-out and overstocked retailers. These studies have the same frequency (once in a period) for replenishments and transshipments. In practice, replenishments are less frequent than transshipments. Transshipment studies usually assume a basestock (order-up-to) replenishment policy. A stark exception is Herer and Rashit (1999), where an $(s, S)$ replenishment policy is shown to be nonoptimal in the presence of fixed and joint replenishment costs. Robinson (1990) provides another exception by showing the stationarity of an optimal basestock policy in a multilocation setting.

For continuous review transshipment models with no fixed cost of replenishment, one-for-one replenishment is a common policy especially for expensive low-demand items. Grahovac and Chakravarty (2001) and Kukreja et al. (2001) both formulate multilocation problems with one-for-one replenishments, where the latter incorporates service level constraints. Continuous review transshipment models with a fixed cost of ordering are scarce in the literature, with the exceptions of Xu et al. (2003) and Minner and Silver (2005). Our model has discrete time. However, we connect it to continuous time when the demands follow independent Poisson processes.

Archibald et al. (1997) is one of the few studies that allow multiple transshipments per cycle in a continuous time model with independent Poisson demands. Archibald and colleagues consider an emergency order instead of backordering when a transshipment is not an option. In this setting, they obtain optimal threshold times (corresponding to hold-back levels) to manage transshipments.

Replenishment time is often assumed to be negligible to simplify the replenishment problem by having the same inventory position and inventory level at each retailer. As exceptions, Tagaras and Cohen (1992) and Tagaras (1999) consider a positive replenishment time. By studying some reasonable policies, the former points out that holding back some inventory at a retailer for forthcoming uncertain demand does not reduce the cost. By studying all policies, our paper shows that holding back inventory is optimal. In addition to negligible replenishment time, the transshipment time is assumed to be negligible in most studies, with the exception of Tagaras and Vlachos (2002), which models preventive transshipments.

Inventory sharing is one of the emergency demand satisfaction methods used to decrease lost sales and/ or backorders. There is also significant work dealing with various other methods including seller-induced downward demand substitution (Hsu et al. 2005, Rao et al. 2004), two supply modes (Moinzadeh and Nahmias 1988), and expediting outstanding orders (Duran et al. 2004).

With respect to the literature, the contributions of our paper can be summarized as follows: (1) We analyze a centrally managed model of two retailers, where replenishment decisions are made by considering multiple transshipments per cycle. Periodically incurred holding and backorder costs, and a positive transshipment time are allowed. Incorporation of these has not been done in the pooling literature. Our model results in an optimal transshipment policy characterized by chronologically nondecreasing holdback levels. These hold-back levels are managerially friendly as they are easy to compute and use. (2) The sensitivity of the transshipment policy to problem parameters, such as demand probabilities, transshipment time, and unit holding and backorder costs, is analytically investigated. (3) The cost savings provided by our optimal transshipment policy (which is a partial pooling policy) over the extreme policies of complete and no pooling are numerically shown. (4) When the replenishment time is positive, transshipment and replenishment policies are intertwined. The difficulty of simultaneously finding these policies is overcome by developing an easily implementable heuristic solution (based on an upper bound [UB] of the cost) that yields only a slightly higher cost (on average $1.1 \%$ ) than the optimal cost.

\section{A Two-Retailer System with Multiple In-Cycle Transshipments}

This section studies a model of two retailers interacting within an inventory sharing system. A dynamic program is formulated in section 3.1 to compute the optimal cost incurred during a cycle. This cost becomes a part of the long-run average cost expression in section 3.2. Then in section 3.3, some structural properties that lead to optimal replenishment and transshipment policies are obtained. 


\subsection{Development of In-Cycle Costs}

Assuming stationary demands and the same daily hours of operation at the retailers, we develop a discrete time model by dividing a cycle into $N$ short decision periods. In this time discretization, the periods are short enough so that there can be at most one unit demand in each period, either at retailer 1 or 2 , or neither. In a period, demands at retailers 1 and 2 happen with probabilities $p_{1}$ and $p_{2}$, respectively, where $p_{1}+p_{2} \leq 1$. The total demand $\left(\xi_{1}^{N}, \xi_{2}^{N}\right)$ per cycle has a multinomial distribution:

$$
\begin{aligned}
P\left(\xi_{1}^{N}=\right. & \left.k_{1}, \xi_{2}^{N}=k_{2} ; N, p_{1}, p_{2}\right)=\frac{N !}{k_{1} ! k_{2} !\left(N-k_{1}-k_{2}\right) !} \\
& \times p_{1}^{k_{1}} p_{2}^{k_{2}}\left(1-p_{1}-p_{2}\right)^{N-k_{1}-k_{2}},
\end{aligned}
$$

which is used as a demand model in Lee and Tang (1998) and Righter and Shanthikumar (2001). It is related to the extensive consumer choice models of marketing, e.g., see Chandukala et al. (2008) and the references therein.

Our demand model can be motivated by considering the demand occurrence process in two steps. In the first step, a unit of customer demand occurs with probability $p_{1}+p_{2}$ in a period. In the second step, the customer goes to retailer 1 or 2 with probabilities $p_{1} /$ $\left(p_{1}+p_{2}\right)$ or $p_{2} /\left(p_{1}+p_{2}\right)$, respectively. Then $N\left(p_{1}+p_{2}\right)$ can be interpreted as the market size while $p_{1} / p_{2}$ is the relative size of retailer 1 with respect to (wrt) retailer 2 . To operationalize our demand model in real-life with historical demand data, we can set the empirical average of demands at retailers 1 and 2 equal to $N p_{1}$ and $N p_{2}$, respectively, and set the empirical correlation of the demands equal to the correlation of the multinomial distribution. This is detailed in the supporting information appendix.

Our demand model is applicable to low-demand (slow-moving) products because it has independent, Poisson demands as a limiting case. If $N p_{1}$ and $N p_{2}$ are kept constant while increasing $N$ and decreasing $p_{1}$ and $p_{2}$, the joint probability $P\left(\xi_{1}^{N}=k_{1}, \xi_{2}^{N}=k_{2} ; N, p_{1}\right.$, $p_{2}$ ) in (1) converges to the product of two Poisson probabilities with rates $N p_{1}$ and $N p_{2}$; see the supporting information appendix. This is actually a common passage to continuous time from discrete time models. See Talluri and van Ryzin (1998), Bitran and Mondschein (1997), Maglaras and Meissner (2006), and Lee and Hersh (1993). To apply our model in a context of independent Poisson demands with rates $\lambda_{1}$ and $\lambda_{2}$ at retailers, it suffices to first set $N$ sufficiently large and then to set $p_{\mathrm{i}}=\lambda_{i} / N$ for $i=1$ and 2 . Time discretization facilitates the analysis to obtain analytical results (Talluri and van Ryzin 1998). For example, Archibald et al. (1997), whose continuous-time transshipment model has Poisson demands, mention on page 178 that their analysis will be harder, if at all possible, with continuously incurred holding costs or a positive replenishment time. In addition, as the continuoustime model computations are generally performed via their discrete-time analogies, even a continuous-time model often requires time discretization before implementation.

A consequence of our demand construct is that the processes are necessarily negatively correlated. However, the model can be used to approximate the case of independent Poisson demands. Despite capturing independent and negatively correlated demands, our demand construct does not directly allow demands at both retailers in the same period. So it cannot be directly used to model positively correlated demands. However, if both retailers have demands in the same period, it is appropriate to presume that the requested retailer always meets her demand before considering a request from the requesting retailer. Thus, two demands in a single period can appear as if they are demands in two consecutive periods. With this reasoning, the formulation changes only slightly (Çömez et al. 2010) in the case of positively correlated demands.

Transshipments are requested after a demand realization at a stocked-out retailer and are processed one by one (not in batches). These features coincide with the transshipment of low-demand products in practice, especially as is typical in automotive transshipments. Applicability of these features of the model are revisited in section 6. Grahovac and Chakravarty (2001) and Zhao et al. (2006) report small or no change in the optimal policy parameters when a transshipment request can be made in advance of a stock-out.

The number of decision periods remaining until the next replenishment is denoted by $n$. In period $n$, if retailers have inventory, they satisfy demand from their own inventory. If one of the retailers has no inventory to satisfy his demand, a transshipment request is sent to the other retailer. Then the following actions are taken according to the transshipment policy.

- If the transshipment request is accepted, a unit is shipped to the requesting retailer. Then the IM incurs transportation cost $K$ per unit. Also during the transshipment time $T$, both backorder cost with rate $\pi$ and holding cost with rate $h_{0}$ per period are incurred. The rate $h_{0}$ can be either the requesting or the requested retailer's holding cost rate or another rate. In summary, cost of a transshipment is $T\left(\pi+h_{0}\right)+K$.

- If the request is rejected, the demand is backordered at the requesting retailer until the next replenishment. Thus the IM incurs a backorder cost of $n \pi$.

- At the end of a period, holding costs $h_{1}$ and $h_{2}$ are, respectively, charged for each unit of on-hand inventory at retailers 1 and 2 . 


\section{Table 1 Notation}

\begin{tabular}{|c|c|}
\hline \multicolumn{2}{|l|}{ Parameters } \\
\hline$i$ & Retailer index, $i \in\{1,2\}$ \\
\hline$n$ & Number of remaining periods until the next replenishment \\
\hline$N$ & Number of periods in a replenishment cycle \\
\hline$m$ & Replenishment cycle index, $m \in\{1, \ldots, M\}$ \\
\hline$p_{i}$ & Probability of a customer demand at retailer $i$ in a period \\
\hline$T$ & Transshipment time between the retailers \\
\hline$L$ & $\begin{array}{l}\text { Replenishment time between the retailers and their manufac- } \\
\text { turer }\end{array}$ \\
\hline$\pi$ & Backorder cost per unit per period \\
\hline$h_{i}$ & Holding cost per unit per period at retailer $i$ \\
\hline$h_{0}$ & Holding cost per unit per period during a transshipment \\
\hline$c$ & Purchasing cost per unit \\
\hline K & Transportation cost per unit transshipped \\
\hline$\xi_{i}^{n}$ & $\begin{array}{l}\text { Random demand realized by retailer } i \text { from customers during } n \\
\text { periods }\end{array}$ \\
\hline \multicolumn{2}{|l|}{ Variables } \\
\hline$x_{i}$ & Inventory level at retailer $i$ at the beginning of a given period \\
\hline$\tau$ & $\begin{array}{l}\text { Transshipment policy, which specifies when transshipment } \\
\text { requests are accepted or not }\end{array}$ \\
\hline$\hat{\xi}_{i}^{n}\left(x_{1}, x_{2} ; \tau\right)$ & $\begin{array}{l}\text { Sum of the demands met and backordered by retailer } i \text {, including } \\
\text { the transshipments to the other retailer, during the next } n \text { periods } \\
\text { when the current inventory levels are }\left(x_{1}, x_{2}\right) \text { and the trans- } \\
\text { shipment policy is } \tau\end{array}$ \\
\hline$y_{i}^{m}$ & Inventory level at retailer $i$ at the time of ordering for cycle $m$ \\
\hline$q_{i}^{m}$ & $\begin{array}{l}\text { Replenishment amount received by retailer } i \text { at the beginning of } \\
\text { cycle } m\end{array}$ \\
\hline$z_{i}^{m}$ & $\begin{array}{l}\text { Inventory position at retailer } i \text { at the time of ordering, } \\
z_{i}^{m}=y_{i}^{m}+q_{i}^{m}\end{array}$ \\
\hline$Z$ & $\begin{array}{l}\text { Vector of }\left[\left(z_{1}^{1}, z_{2}^{1}\right),\left(z_{1}^{2}, z_{2}^{2}\right), \ldots\right] \text { denoting the replenishment } \\
\text { policy at the retailers for the planning horizon }\end{array}$ \\
\hline$V_{n}\left(x_{1}, x_{2}\right)$ & $\begin{array}{l}\text { Minimum expected total cost for the remaining } n \text { periods, when } \\
\text { current inventory levels are }\left(x_{1}, x_{2}\right)\end{array}$ \\
\hline$W_{m}\left(y_{1}^{1}, y_{2}^{1} \mid Z\right)$ & $\begin{array}{l}\text { Minimum expected total cost for } m \text { cycles, when the initial } \\
\text { inventories are }\left(y_{1}^{1}, y_{2}^{1}\right) \text { and the replenishment policy is } Z\end{array}$ \\
\hline
\end{tabular}

These costs are incurred by the IM in each period until the end of a cycle. Let $\mathscr{N}=\{1,2, \ldots\}$ and $\mathscr{N}^{-}=\{0,-1,-2, \ldots\}$ be the sets of positive and nonpositive integers. The notation used throughout the paper is summarized in Table 1.

The inventory level at a retailer is the on-hand inventory minus the backorders. The minimum expected total cost $V_{n}\left(x_{1}, x_{2}\right)$ achievable in the remaining $n$ periods is computed recursively in each of the following three cases. First, if each retailer has at least one unit of inventory, each retailer satisfies his own demand. $V_{n}\left(x_{1}, x_{2}\right)$ can be written as

$$
\begin{aligned}
V_{n}\left(x_{1}, x_{2}\right)= & p_{1}\left[V_{n-1}\left(x_{1}-1, x_{2}\right)+h_{1}\left(x_{1}-1\right)+h_{2} x_{2}\right] \\
& +p_{2}\left[V_{n-1}\left(x_{1}, x_{2}-1\right)+h_{1} x_{1}+h_{2}\left(x_{2}-1\right)\right] \\
& +\left(1-p_{1}-p_{2}\right)\left[V_{n-1}\left(x_{1}, x_{2}\right)+h_{1} x_{1}+h_{2} x_{2}\right] \\
& x_{1}, x_{2} \in \mathscr{N}
\end{aligned}
$$

In this case, no transshipment request is made and the IM incurs holding costs based on the remaining inventories.

Similarly, there is no transshipment decision to make when both retailers are stocked-out. Then the IM incurs costs for backorders:

$$
\begin{aligned}
V_{n}\left(x_{1}, x_{2}\right)= & p_{1}\left[n \pi+V_{n-1}\left(x_{1}-1, x_{2}\right)\right] \\
& +p_{2}\left[n \pi+V_{n-1}\left(x_{1}, x_{2}-1\right)\right] \\
& +\left(1-p_{1}-p_{2}\right) V_{n-1}\left(x_{1}, x_{2}\right), \\
& x_{1}, x_{2} \in \mathscr{N}^{-} .
\end{aligned}
$$

When an item is backordered in period $n$, its entire backorder $\operatorname{cost} n \pi$ is charged at once in period $n$. That is, the backorder cost is charged item by item. Our model allows retailers to request a transshipment to meet their current demand, not for already backordered demands.

The IM makes transshipment decisions only in the third case, when a retailer is stocked-out while the other still has inventory. Without loss of generality, the stocked-out retailer is called retailer 2. Each time a customer arrives at stocked-out retailer 2, a transshipment request is sent to retailer 1 . If retailer 1 accepts the request according to the IM's instructions, her inventory immediately reduces by one and backorder cost is incurred until the transshipment arrives at retailer 2. Otherwise, retailer 2's inventory level reduces by one and the backorder cost is incurred until the next replenishment. Formally,

$$
\begin{aligned}
V_{n}\left(x_{1}, x_{2}\right)= & p_{1}\left[V_{n-1}\left(x_{1}-1, x_{2}\right)+h_{1}\left(x_{1}-1\right)\right] \\
& +p_{2}\left[\operatorname { m i n } \left\{n \pi+V_{n-1}\left(x_{1}, x_{2}-1\right)+h_{1} x_{1},\right.\right. \\
T\left(\pi+h_{0}\right) & \left.\left.+K+V_{n-1}\left(x_{1}-1, x_{2}\right)+h_{1}\left(x_{1}-1\right)\right\}\right] \\
& +\left(1-p_{1}-p_{2}\right)\left[V_{n-1}\left(x_{1}, x_{2}\right)+h_{1} x_{1}\right], \\
& x_{1} \in \mathscr{N}, x_{2} \in \mathscr{N}^{-} .
\end{aligned}
$$

When a unit is transshipped from retailer 1 to retailer 2 for a customer arriving in period $n$, the unit is taken out of retailer 1 inventory in that period and is assigned to the customer. The backorder and holding costs during $T$ are immediately charged to the cost function in period $n$. The transshipped unit never enters into retailer 2 inventory. If retailer 1 stocks-out before retailer $2, V_{n}\left(x_{1}, x_{2}\right)$ can be written analogous to (4). Note that (4) does not impose a transshipment policy on retailer 1 . Indeed, this policy is one of our results.

At the end of a cycle, where $n=0$,

$$
V_{0}\left(x_{1}, x_{2}\right)=0, \quad x_{1}, x_{2} \in \mathscr{N} \cup \mathscr{N}^{-} .
$$

This completes the formulation of the transshipment problem. The minimum total inventory holding 
and backorder costs incurred over a cycle is $V_{N}\left(z_{1}, z_{2}\right)$ when the cycle starts with inventories $z_{1}$ and $z_{2}$.

REMARK 1. Our model can also be useful to a single retailer selling two products that are substitutable after some reconfiguration. The retailer may receive a customer demand in each period for products 1 or 2 , with probabilities $p_{1}$ and $p_{2}$, respectively. When product 2 stocks-out, a product 2 demand can be satisfied by reconfiguring a product 1 into a product 2 . This reconfiguration is analogous to a transshipment from retailer 1 to retailer 2 . Such reconfiguration occurs at a car dealer that sells the same type of a car with different but easily reconfigurable options such as cassetteCD player, SUV seat arrangement, and spoiler.

\subsection{Development of Long-Run Average Cost}

Retailer inventories are replenished at the beginning of each cycle. The fixed ordering costs for replenishments are taken to be zero, because they are irrelevant for our problem, where the replenishment schedule is prespecified. Initially the problem is studied with zero replenishment time $(L=0)$. Later in section 5 , a positive replenishment time is incorporated. The accompanying challenges are discussed along with an effective heuristic to address them.

To obtain the expected value of long-run average cost, the expected total cost is defined first for $M$ cycles. Averaging the expected total cost when $M$ goes to infinity results in the expected long-run average cost. The inventory levels of retailers at the time of ordering for cycle $m$ are denoted as $\left(y_{1}^{m}, y_{2}^{m}\right)$. The IM replenishes retailer inventories such that $\left(z_{1}^{m}, z_{2}^{m}\right)$ are the inventory positions immediately after ordering for cycle $m$, where inventory position is defined as the on-hand inventory minus backorders plus the outstanding replenishment order. The replenishment policy for all cycles in the planning horizon is denoted as a vector $Z=\left[\left(z_{1}^{1}, z_{2}^{1}\right),\left(z_{1}^{2}, z_{2}^{2}\right), \ldots\right]$. The replenishment order amounts for cycle $m$ are $\left(q_{1}^{m}, q_{2}^{m}\right)$, where $q_{i}^{m}=z_{i}^{m}-y_{i}^{m}$ for $i \in\{1,2\}$.

After inventories are replenished, demand is met during the cycle by possibly transshipping inventory between retailers. This results in total cycle cost $V_{N}\left(z_{1}^{m}, z_{2}^{m}\right)$ for cycle $m$. $W_{M}\left(y_{1}^{1}, y_{2}^{1} \mid Z\right)$ is the expected total cost for an $M$-cycle problem with initial inventory levels $\left(y_{1}^{1}, y_{2}^{1}\right)$ when the ordering policy $Z$ is used

$$
\begin{aligned}
& W_{M}\left(y_{1}^{1}, y_{2}^{1} \mid Z\right) \\
& =E\left[\sum_{m=1}^{M}\left[c\left(z_{1}^{m}+z_{2}^{m}-y_{1}^{m}-y_{2}^{m}\right)+V_{N}\left(z_{1}^{m}, z_{2}^{m}\right)\right]\right],
\end{aligned}
$$

which should be appended with the constraint $z_{i}^{m} \geq$ $y_{i}^{m}$ to restrict the inventory positions at ordering to be greater than or equal to the inventory levels before ordering. However, to avoid the difficulties of constraints, we use a relaxation trick. We show that optimal inventory positions for the average cost are stationary from one cycle to the next. Thus inventory levels cannot surpass optimal inventory positions once they drop below optimal inventory positions. While inventory levels are below the optimal positions, order quantities remain non-negative. Note that the cost of not ordering replenishments in some initial consecutive cycles is finite. Then the IM can without loss of generality bring inventory levels below the optimal inventory positions. This finite cost does not affect the average cost. A similar relaxation is used by Zheng (1994) to prove the optimality of an ordering policy in a different context. However, these relaxation ideas do not work for the total discounted cost over an infinite horizon (Presman and Sethi 2006).

Let $\xi_{i}^{N}$ be the random demand realized by retailer $i$ from customers during a cycle of $N$ periods, which is binomially distributed with $N$ and $p_{i}$ for $i \in\{1,2\}$. $\hat{\xi}_{i}^{N}\left(z_{1}, z_{2} ; \tau\right)$ is defined as the demand met and backordered by retailer $i$ during a cycle, which starts with inventory positions $\left(z_{1}, z_{2}\right)$. If no retailer stocks-out, $\hat{\xi}_{i}^{N}\left(z_{1}, z_{2} ; \tau\right) \stackrel{d}{=} \xi_{i}^{N}$, where "d $=$ " between two random variables indicates equality of the random variables in distribution. If retailer 2 stocks-out, retailer 1 may meet some of retailer 2's demand. If so, then $\hat{\xi}_{1}^{N}\left(z_{1}, z_{2} ; \tau\right)$ is stochastically larger than $\xi_{1}^{N}$. Since the sum of the demands met and backordered by the retailers is equal to the total demand arriving into the system, $\hat{\xi}_{1}^{N}\left(z_{1}, z_{2} ; \tau\right)+\hat{\xi}_{2}^{N}\left(z_{1}, z_{2} ; \tau\right) \stackrel{d}{=} \xi_{1}^{N}+\xi_{2}^{N}$, which is also binomially distributed with $N$ and $p_{1}+p_{2}$. Note that this sum is independent of $z_{1}, z_{2}$, and $\tau$.

The inventory levels at the time of ordering for any cycle $m+1$ can be calculated as $y_{i}^{m+1}=z_{i}^{m}-\hat{\xi}_{i}^{N}\left(z_{1}^{m}, z_{2}^{m}\right.$; $\tau)$ for $i \in\{1,2\}$. Following the definitions of $y_{1}^{m}$ and $y_{2}^{m}$,

(6) can be rewritten as

$$
\begin{array}{rl} 
& W_{M}\left(y_{1}^{1}, y_{2}^{1} \mid Z\right) \\
=E & E\left[\sum_{m=1}^{M}\left[V_{N}\left(z_{1}^{m}, z_{2}^{m}\right)\right]+c\left[z_{1}^{1}+z_{2}^{1}-y_{1}^{1}-y_{2}^{1}\right]\right. \\
& +c\left[z_{1}^{2}+z_{2}^{2}-z_{1}^{1}+\hat{\xi}_{1}^{N}\left(z_{1}^{1}, z_{2}^{1} ; \tau\right)-z_{2}^{1}+\hat{\xi}_{2}^{N}\left(z_{1}^{1}, z_{2}^{1} ; \tau\right)\right] \\
& +\cdots+c\left[z_{1}^{M}+z_{2}^{M}-z_{1}^{M-1}+\hat{\xi}_{1}^{N}\left(z_{1}^{M-1}, z_{2}^{M-1} ; \tau\right)-z_{2}^{M-1}\right. \\
& \left.\left.+\hat{\xi}_{2}^{N}\left(z_{1}^{M-1}, z_{2}^{M-1} ; \tau\right)\right]\right] \\
= & E\left[\sum_{m=1}^{M} V_{N}\left(z_{1}^{m}, z_{2}^{m}\right)+\sum_{m=1}^{M-1} c\left(\hat{\xi}_{1}^{N}\left(z_{1}^{m}, z_{2}^{m} ; \tau\right)\right.\right. \\
& \left.\left.+\hat{\xi}_{2}^{N}\left(z_{1}^{m}, z_{2}^{m} ; \tau\right)\right)+c\left(z_{1}^{M}+z_{2}^{M}-y_{1}^{1}-y_{2}^{1}\right)\right] .
\end{array}
$$


Our objective is to minimize the expected average cost over an infinite number of cycles

$$
\min _{Z} \lim _{M \rightarrow \infty} \frac{W_{M}\left(y_{1}^{1}, y_{2}^{1} \mid Z\right)}{M} .
$$

Since $\hat{\xi}_{1}^{N}\left(z_{1}, z_{2} ; \tau\right)+\hat{\xi}_{2}^{N}\left(z_{1}, z_{2} ; \tau\right) \stackrel{d}{=} \xi_{1}^{N}+\xi_{2}^{N}$, which is independent of $\tau$ and $Z$, it can be omitted when plugging the definition of $W_{M}\left(y_{1}^{1}, y_{2}^{1} \mid Z\right)$ from (7) into (8). Thus the long-run average cost becomes

$\min _{Z}\left\{\lim _{M \rightarrow \infty} \frac{\sum_{m=1}^{M} V_{N}\left(z_{1}^{m}, z_{2}^{m}\right)}{M}+\lim _{M \rightarrow \infty} \frac{c\left(z_{1}^{M}+z_{2}^{M}-y_{1}^{1}-y_{2}^{1}\right)}{M}\right\}$

$$
\begin{aligned}
& =\min _{Z} \lim _{M \rightarrow \infty} \frac{\sum_{m=1}^{M} V_{N}\left(z_{1}^{m}, z_{2}^{m}\right)}{M} \geq \lim _{M \rightarrow \infty} \frac{\sum_{m=1}^{M} V_{N}\left(z_{1}^{*}, z_{2}^{*}\right)}{M} \\
& =\min _{z_{1}, z_{2}} V_{N}\left(z_{1}, z_{2}\right) .
\end{aligned}
$$

The second term in (9) becomes 0 as $M \rightarrow \infty$ because $z_{1}^{M}, z_{2}^{M}, y_{1}^{1}$, and $y_{2}^{1}$ are all finite. $\left(z_{1}^{*}, z_{2}^{*}\right)$ are optimal inventory replenishment positions obtained by minimizing $V_{N}\left(z_{1}, z_{2}\right)$. Although $\min _{z_{1}, z_{2}} V_{N}\left(z_{1}, z_{2}\right)$ is a lower bound for the long-run average cost, since the policy $Z=\left(z_{1}^{1}=\right.$ $\left.z_{1}^{*}, z_{2}^{1}=z_{2}^{*}, z_{1}^{2}=z_{1}^{*}, z_{2}^{2}=z_{2}^{*}, \ldots\right)$ is feasible, $V_{N}\left(z_{1}^{*}, z_{2}^{*}\right)$ is exactly equal to the minimum average cost. In summary, inventory positions that minimize the holding, backorder, and transshipment costs over a single cycle also minimize the expected long-run average cost.

REMARK 2. In view of (10), the optimal values of replenishment levels are independent of the purchasing cost when the objective function is average cost. On the contrary, such independence does not hold when the first cycle cost $c\left(z_{1}^{1}+z_{2}^{1}-y_{1}^{1}-y_{2}^{1}\right)+V_{N}\left(z_{1}^{1}, z_{2}^{1}\right)$ is minimized. Hence, intuition obtained from a singlecycle problem unfortunately is not valid for the average cost problem. In particular, the optimal replenishment amounts do not decrease as the purchase cost increases in the average cost problem. The sum of the replenishment amounts at the end of a cycle must be equal to the total demand observed in that cycle. If this sum is always less (respectively, more) than total demand, the inventory levels $y_{1}^{m}$ and $y_{2}^{m}$ will approach negative (respectively, positive) infinity. Inventory levels of negative or positive infinity are clearly neither practical nor stable nor optimal.

REMARK 3. The objective function expression changes significantly when the purchase costs of retailers 1 and 2 differ. Denoting these costs by $c_{1}$ and $c_{2}$, the $M$-cycle total cost becomes $W_{M}\left(y_{1}^{1}, y_{2}^{1} \mid Z\right)=$
$E\left[\sum_{m=1}^{M}\left[c_{1}\left(z_{1}^{m}-y_{1}^{m}\right)+c_{2}\left(z_{2}^{m}-y_{2}^{m}\right)+V_{N}\left(z_{1}^{m}, z_{2}^{m}\right)\right]\right] . \quad$ In this expression, $c_{1}\left(z_{1}^{m}-y_{1}^{m}\right)+c_{2}\left(z_{2}^{m}-y_{2}^{m}\right)$ cannot be written in general as a function of the total random demand $\xi_{1}^{N}+\xi_{2}^{N}$. Thus the objective function would be more complicated than (7) when $c_{1} \neq c_{2}$.

When $c_{1}=c_{2}=c$, the simpler objective in (10) allows us to focus on the transshipment problem. Since neither the replenishment nor transshipment decisions are affected by the purchase cost, $c$ can be set equal to zero for the remainder of the paper.

\subsection{Optimal Transshipment and Replenishment Policies}

The inventory positions $\left(z_{1}, z_{2}\right)$ depend on transshipment decisions. But the reverse is not true. A transshipment decision depends only on the current inventory levels $\left(x_{1}, x_{2}\right)$, but does not depend on $\left(z_{1}, z_{2}\right)$ once $\left(x_{1}, x_{2}\right)$ are given. This robustness facilitates not only the analysis of the transshipment problem but also the implementation of the optimal transshipment policy.

A transshipment request happens only when one of the retailers has inventory and the other does not. Continuing with our convention of naming the retailer with inventory as retailer 1 , the inventory levels belong to $\left\{\left(x_{1}, x_{2}\right): x_{1} \in \mathscr{N}, x_{2} \in \mathscr{N}^{-}\right\}$when a transshipment happens. Whether inventory levels ever belong to this set during a cycle is a consequence of demand realizations. For example, the demands can be so low in a cycle that inventories stay positive and no transshipment is required.

The IM deals with the trade-off represented by the minimum in (4). To better understand this trade-off, we set $\delta_{n}\left(x_{1}\right):=V_{n}\left(x_{1}-1, x_{2}\right)-V_{n}\left(x_{1}, x_{2}\right)$ for $x_{1} \in \mathscr{N}$ and $x_{2} \in \mathscr{N}^{-} ; \delta_{n}\left(x_{1}\right)$ is the marginal benefit of one extra unit of inventory at retailer 1 . The marginal benefit is based only on the inventory at retailer 1 . For an intuitive explanation of this, note that the cost $V_{n}\left(x_{1}, x_{2}\right)$ is independent of the number of units backordered at retailer 2 for two reasons. First, the backorder cost is charged at once when a unit is backordered. Second, a backordered demand remains backordered in a cycle. Thus $V_{n}$ does not capture any backorder costs for the units backordered earlier than period $n$. So the number of already backordered units does not affect $V_{n}$. As a result, $V_{n}\left(x_{1}, x_{2}-1\right)=V_{n}\left(x_{1}, x_{2}\right)$ for $x_{2}<0$, so the marginal cost of one more backorder at retailer 2 is zero. A formal justification is given by Lemma 1.

\section{LEMMA 1.}

(i) $V_{n}\left(x_{1}, x_{2}\right)=V_{n}\left(x_{1}, x_{2}^{\prime}\right)$ for $x_{2}, x_{2}^{\prime} \in \mathscr{N}^{-}$.

(ii) $V_{n}\left(x_{1}, x_{2}\right)=V_{n}\left(x_{1}^{\prime}, x_{2}^{\prime}\right)$ for $x_{1}, x_{2}, x_{1}^{\prime}, x_{2}^{\prime} \in \mathscr{N}^{-}$. Consequently,

$$
\begin{aligned}
V_{n}\left(x_{1}, x_{2}\right)= & V_{n}(0,0) \\
= & \left(p_{1}+p_{2}\right) \frac{n(n+1)}{2} \pi \\
& \quad \text { for } \quad x_{1}, x_{2} \in \mathscr{N}^{-} .
\end{aligned}
$$


By definition, $\delta_{0}\left(x_{1}\right)=0$ for every $x_{1}$ and $\delta_{n}\left(x_{1}\right)$ can be written recursively as

$$
\begin{aligned}
& \delta_{n}\left(x_{1}\right)=-h_{1}+\left(1-p_{1}\right) \delta_{n-1}\left(x_{1}\right)+p_{1} \delta_{n-1}\left(x_{1}-1\right) \\
& +p_{2}\left[\min \left\{n \pi+h_{1}, T\left(\pi+h_{0}\right)+K+\delta_{n-1}\left(x_{1}-1\right)\right\}\right. \\
& \left.-\min \left\{n \pi+h_{1}, T\left(\pi+h_{0}\right)+K+\delta_{n-1}\left(x_{1}\right)\right\}\right], \\
& x_{1} \geq 2, x_{2} \in N^{-} .
\end{aligned}
$$

We must separately write $\delta_{n}\left(x_{1}=1\right)$, which includes the total cost $V_{n}\left(0, x_{2}\right)$ that has a different form from the general $V_{n}\left(x_{1}, x_{2}\right)$; see (4) and (5).

$$
\begin{aligned}
& \delta_{n}\left(x_{1}=1\right)= \\
& -h_{1}+\left(1-p_{1}\right) \delta_{n-1}\left(x_{1}=1\right)+\left(p_{1}+p_{2}\right)\left(n \pi+h_{1}\right) \\
& -p_{2} \min \left\{n \pi+h_{1}, T\left(\pi+h_{0}\right)+K+\delta_{n-1}\left(x_{1}=1\right)\right\} .
\end{aligned}
$$

The minima in (11) and (12) represent the trade-off involved in accepting a transshipment request. The request is accepted if and only if $\delta_{n-1}\left(x_{1}\right) \leq$ $(n-T) \pi+h_{1}-T h_{0}-K$. We refer to $\delta_{n-1}\left(x_{1}\right)$ and $(n-T) \pi+h_{1}-T h_{0}-K$, respectively, as the marginal benefit and marginal cost of rejecting a request. The monotonic behavior of the marginal benefit function with regard to (wrt) the change in inventory level $x_{1}$ is important to establish the structure of a transshipment policy.

\section{LEMMA 2.}

(i) The marginal benefit is non-increasing in inventory: $\delta_{n}\left(x_{1}\right) \leq \delta_{n}\left(x_{1}-1\right), x_{1} \in \mathscr{N}$.

(ii) The marginal benefit cannot be more than the cost of backordering a unit until the end of the cycle: $\delta_{n}\left(x_{1}\right) \leq n \pi$.

(iii) The marginal benefit of infinite amount of inventory is the holding cost paid until the end of the cycle: $\lim _{x_{1} \rightarrow \infty} \delta_{n}\left(x_{1}\right)=-n h_{1}$.

Lemma 2(i) says that the marginal benefit of inventory decreases or stays the same as the inventory level rises. Parts (ii) and (iii) of the lemma in conjunction with (i) establish the upper and lower limits for the marginal benefit, respectively. Both upper and lower limits are linear in the number of remaining periods $n$. Combining Lemma 2(i) with the fact that a transshipment request is accepted if $\delta_{n-1}\left(x_{1}\right) \leq(n-T) \pi$ $+h_{1}-T h_{0}-K$, we conclude that if the transshipment request is accepted with inventory $x_{1}$, it must also be accepted with inventory $x_{1}^{\prime} \geq x_{1}$. Similarly, if the transshipment request is rejected with inventory $x_{1}$, it must also be rejected with inventory $x_{1}^{\prime \prime} \leq x_{1}$. These observations lead to the optimal transshipment policy in Theorem 1(i).

\section{THEOREM 1.}

(i) For each period $n$, there exists a hold-back level $\tilde{x}_{1}^{n}$ for retailer 1 such that it is optimal to reject (respectively, accept) the transshipment request when $x_{1} \leq \tilde{x}_{1}^{n}$ (respectively, $x_{1}>\tilde{x}_{1}^{n}$ ).

(ii) The hold-back level is finite, i.e., partial pooling is viable if $n \geq\left(T\left(\pi+h_{0}\right)+K\right) /\left(\pi+h_{1}\right)$.

The hold-back level can be found from $\tilde{x}_{1}^{n}:=$ $\max \left\{x \in \mathscr{N}: \delta_{n-1}\left(x_{1}\right)>(n-T) \pi+h_{1}-T h_{0}-K\right\}$. The sequence of hold-back levels $\left\{\tilde{x}_{1}^{n}: 1 \leq n \leq N\right\}$ is the optimal transshipment policy $\tau^{*}$ for retailer 1 . From $\delta_{0}(\cdot)=0,(11)$, and (12), a hold-back level is independent of the cycle length $N$ and the beginning inventory levels in a cycle. If $\delta_{n-1}(1) \leq(n-T) \pi+$ $h_{1}-T h_{0}-K$, then $\tilde{x}_{1}^{n}=0$, i.e., complete pooling is optimal when the benefit of retailer 1 keeping one unit of inventory is sufficiently low. At the other extreme, no pooling is optimal when the number of remaining periods is sufficiently small expressed by the inequality as in Theorem 1(ii). To appreciate the inequality better, suppose temporarily that $h_{0}=h_{1}$. Then the inequality becomes $n \geq T+K /\left(\pi+h_{1}\right)$. That is, no transshipment is made if there are fewer than $T+$ $K /\left(\pi+h_{1}\right)$ periods until the next replenishment.

An interesting question is whether retailer 1 holds back more or less inventory early in a cycle. On the one hand, retailer 1 may hold back more inventory early in the cycle to meet more of her demand later in the cycle. On the other hand, backorder costs and the holding cost savings that can be achieved with a transshipment are higher early in the cycle. Thus there are reasons for both low and high hold-back levels early in the cycle. In comparing $\tilde{x}_{1}^{n}$ and $\tilde{x}_{1}^{n-1}$, monotonicity of $\delta_{n}\left(x_{1}\right)$ in $n$ can be useful. However, monotonicity in $n$ does not necessarily hold. What holds is a slightly relaxed monotonicity condition $\delta_{n}\left(x_{1}\right)-\delta_{n-1}\left(x_{1}\right) \leq \pi$, proved in Lemma 3 .

LEMMA 3. The marginal benefit cannot increase more than $\pi$ when the number of remaining periods goes up by 1 : $\delta_{n}\left(x_{1}\right) \leq \delta_{n-1}\left(x_{1}\right)+\pi$ for $n, x_{1} \in \mathscr{N}$.

To motivate the condition, consider two separate cases of having an extra unit of inventory, in periods $n$ and $n+1$. Having the extra unit of inventory early, one can save at most $\pi$, the backorder cost of a unit for one period. If the demand is low, having an extra unit earlier can result in additional holding costs. Although having an extra unit earlier can increase costs, it cannot decrease them more than $\pi$. Thus, the marginal benefit grows by at most $\pi$.

Since the marginal cost $(n-T) \pi+h_{1}-T h_{0}-K$ increases at rate $\pi$, the rate of change in marginal cost is always larger than or equal to the rate of change in marginal benefit. Therefore, if the marginal cost at a particular inventory level is below the marginal benefit in period $n$, it remains below until the end of the cycle. Recall that $\delta_{n}\left(x_{1}\right)-\delta_{n-1}\left(x_{1}\right) \leq \pi$ and that a request is accepted when $\delta_{n-1}\left(x_{1}\right) \leq(n-T) \pi+h_{1}-$ $T h_{0}-K$. These imply that $\delta_{n}\left(x_{1}\right) \leq(n+1-T) \pi+h_{1}-$ 
$T h_{0}-K$. Hence, if a request is accepted with $x_{1}$ units of inventory and with $n$ periods remaining, it is also accepted with the same amount of inventory when $n+1$ periods remain. This gives the next theorem.

THEOREM 2. The hold-back level is nonincreasing in the remaining number of periods: $\tilde{x}_{1}^{n+1} \leq \tilde{x}_{1}^{n}$.

Following the optimal transshipment policy, if a retailer's inventory level in period $n$ is less than or equal to the hold-back level in $n$, it remains less than or equal to the hold-back until the next replenishment. Let $\mathscr{T}$ be the remaining period index $n$ when the inventory at retailer 1 drops to her hold-back level for the first time in a cycle. Then the retailer accepts requests in periods $n \in\{\mathscr{T}+1, \ldots, N\}$ and rejects them in periods $n \in\{1, \ldots, \mathscr{T}\} . \mathscr{T}$ is a stopping time wrt the information set generated by the demands. Existence of a stopping time, which splits a cycle into acceptance and rejection periods, facilitates implementation of our optimal hold-back level policy.

Our model is defined to allow a transshipment request by a retailer for his current demand, not for a backorder. The optimal policy with stopping time $\mathscr{T}$ supports this model because once a retailer rejects a transshipment request, all following requests will be rejected under the optimal policy. Thus, there is no opportunity for a backordered demand to be satisfied with a transshipment later in the cycle. A formal argument can be made first by redefining the value function $V_{n}$ to allow transshipments for backorders and then by showing that no such transshipments happen in the optimal solution; details are in the supporting information appendix.

The choice of the number $N$ of periods is not critical in the implementation of the transshipment policy. This is illustrated in Figures 1 and 2 for the hold-back levels at retailer 1 for a problem with $N=22$ and $N=88$, respectively. When $N$ is increased fourfold, per period costs and demand probabilities are decreased and the transshipment time is increased

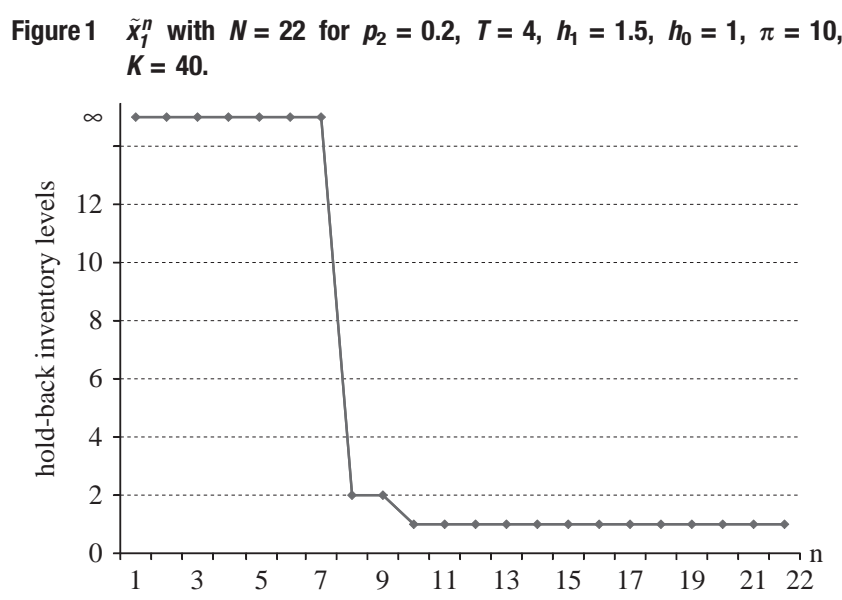

Figure $2 \tilde{x}_{1}^{n}$ with $N=88$ for $p_{2}=0.05, T=16, h_{1}=0.375, h_{0}=0.25$, $\pi=2.5, K=40$.

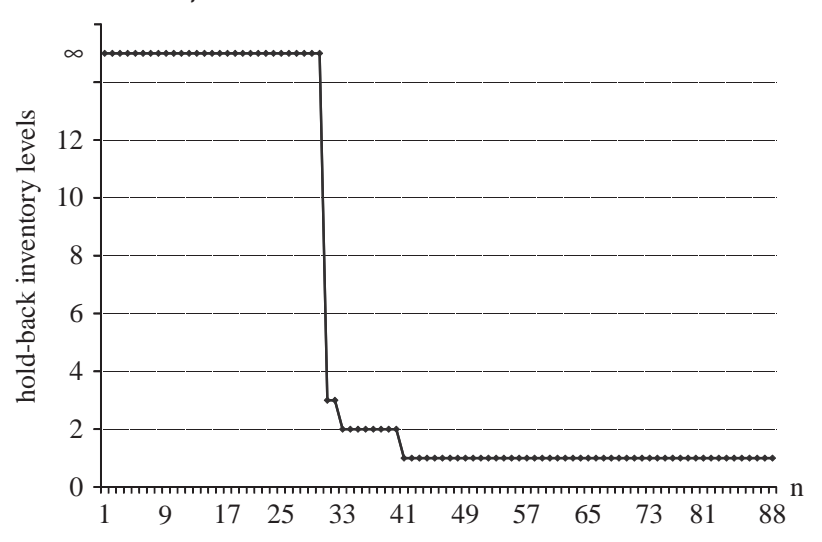

proportionally. Specifically, in Figure $2, p_{1}, p_{2}, h_{1}, h_{0}$, and $\pi$ are reduced by a factor of four and $T$ is quadrupled wrt the corresponding values in Figure 1. These figures illustrate that the pattern of hold-back levels $\tilde{x}_{i}^{n}$ does not change much with $N$. For example, $\tilde{x}_{1}^{n}=2$ for about $32 \leq n \leq 40$ when $N=88$. When $N=88 / 4=22, \quad \tilde{x}_{1}^{n}=2$ for about $8=32 / 4 \leq n<40 /$ $4=10$.

When fewer hold-back levels are desirable in implementation, the hold-back levels can be averaged over certain periods. For example, if replenishments occur monthly and $N=88$ (22 days/month and 4 periods/day), the number of different hold-back levels can be reduced to four: for the first week (periods 6788) in the cycle, the hold-back level can be set equal to the average of $\left\{\tilde{x}_{67}^{n}, \ldots, \tilde{x}_{88}^{n}\right\}$. For the second week, it can be set equal to the average of $\left\{\tilde{x}_{45}^{n}, \ldots, \tilde{x}_{66}^{n}\right\}$ and so on.

The nonincreasing property of our hold-back levels differs from that of Archibald et al. (1997), who propose threshold transshipment times to manage transshipments. These times can be transformed into hold-back levels, which turn out to be nondecreasing in the remaining time. Although this is opposite of our result in Theorem 2, it is not a contradiction because our model setting significantly differs from the model setting in Archibald et al. (1997), both in the treatment of stock-outs and in the cost accounting of on-hand inventory. First, while Archibald and colleagues use emergency orders to meet unsatisfied demand and model lost sales, we backorder unsatisfied demand. Second, in our model, the IM is charged the holding cost for the inventory held at the end of each period, which can be called periodic holding cost accounting (PHA). Archibald and colleagues assess the holding cost only once in a cycle for the inventory held at the end of the cycle, which can be called end of cycle holding cost accounting (EHA). Two critical attributes of our model are (backorder, PHA) while these attributes are (lost sales, EHA) in Archibald 
et al. (1997). With PHA, holding cost computations are more accurate, so PHA is more suitable when $h$ or $N$ is relatively large. On the other hand, EHA computations are slightly easier.

Çömez et al. (2011) compare the (backorder, PHA) model of this paper to a (backorder, EHA) model. There, a hold-back level-based transshipment policy remains optimal with EHA but the monotonicity of the hold-back levels in Theorem 2 does not hold. We expect that a hold-back transshipment policy may remain optimal also for the (lost sales, PHA) model but the monotonicity of the hold-back levels may not. In summary, the (backorder, PHA) model of this paper and the (lost sales, EHA) model in Archibald et al. (1997) can be thought of as two extremes while (backorder, EHA) and (lost sales, PHA) are intermediate models between the extreme models. The hold-back levels are monotone in the extreme models but not in the intermediate models. Moreover, the monotonicity properties in the extreme models are opposites of each other.

The hold-back level-based transshipment policy obtained is similar to the threshold inventory rationing policy used in the literature to model the fulfilment of demand from multiple customer classes. Deshpande et al. (2003) assume static threshold levels for two classes such that when the inventory level drops below the threshold level of a demand class, demands from that class are not satisfied, but backordered. Arslan et al. (2007) study a similar threshold model for more than two classes. Following multiple demand class models, Zhao et al. (2005) define constant threshold levels to manage transshipments at two independent retailers. In contrast, in this study, we show that the optimal transshipment policy for retailers is based on optimal hold-back levels, which change in each decision period depending on the state of the inventory system.

Having the optimal transshipment policy developed, to complete the analysis of the replenishment problem in (10), convexity of cost $V_{n}(\cdot, \cdot)$ is important. This is established by Lemma 4 .

\section{Lemma 4. $V_{n}\left(z_{1}, z_{2}\right)$ is convex for $z_{1}, z_{2} \in \mathscr{N}$.}

Convexity of the cost function implies the optimality of the basestock policy for the replenishment decision. It also facilitates the search for the optimal basestock values $z_{1}^{*}$ and $z_{2}^{*}$ from the first-order optimality conditions.

REMARK 4 . If the backorder costs are retailer specific, i.e., $\pi_{1} \neq \pi_{2}$, then the hold-back level-based transshipment policies still remain optimal. However, Lemma 3 needs to be relaxed to say that the marginal benefit cannot increase more than $\max \left\{\pi_{1}, \pi_{2}\right\}$ when the number of remaining periods goes up by 1 . This relaxation of Lemma 3 keeps us from generalizing Theorem 2 for $\pi_{1} \neq \pi_{2}$. Other than this remark, $\pi=$ $\pi_{1}=\pi_{2}$ throughout the paper.

\section{Sensitivity and Performance Analyses of Optimal Policies}

This section investigates the sensitivity of hold-back levels wrt problem parameters and improvements in cost provided by the optimal partial pooling policy.

\subsection{Impact of Parameters on Hold-back Levels}

The sensitivity of the hold-back levels for retailer 1 wrt parameters $T, p_{1}, p_{2}, h_{1}, h_{0}$, and $K$ is investigated. Recall that $\tilde{x}_{1}^{n}=\max \left\{x \in \mathscr{N}: \delta_{n-1}\left(x_{1}\right)>(n-T) \pi+h_{1}\right.$ $\left.-T h_{0}-K\right\}$ and the sensitivity of the marginal cost $(n-T) \pi+h_{1}-T h_{0}-K$ to problem parameters is straightforward. Thus a substantial but intermediate step is studying the sensitivity of the marginal benefit. For this purpose, the marginal benefit expression is appended with the parameter under consideration. For example, $\delta_{n}\left(x_{1} ; T\right)$ and $\delta_{n}\left(x_{1} ; p_{1}\right)$ are the marginal benefits, respectively, when the transshipment time is $T$ and the probability of a customer arrival at retailer 1 is $p_{1}$. Sensitivity of $\delta_{n}\left(x_{1} ; T\right), \delta_{n}\left(x_{1} ; p_{1}\right), \delta_{n}\left(x_{1} ; p_{2}\right)$, $\delta_{n}\left(x_{1} ; h_{1}\right), \delta_{n}\left(x_{1} ; h_{0}\right)$, and $\delta_{n}\left(x_{1} ; K\right)$ wrt $T, p_{1}, p_{2}, h_{1}, h_{0}$, and $K$ respectively, is studied in Lemma 5.

$$
\begin{aligned}
& \text { LEMMA 5. For a small } \varepsilon>0 \text {, (i) } \delta_{n}\left(x_{1} ; T+\varepsilon\right)-\delta_{n}\left(x_{1} ; T\right) \geq \\
& -\varepsilon\left(\pi+h_{0}\right) \text {, (ii) } \delta_{n}\left(x_{1} ; p_{1}+\varepsilon\right)-\delta_{n}\left(x_{1} ; p_{1}\right) \geq 0 \text {, (iii) } \delta_{n}\left(x_{1} ; p_{2}+\varepsilon\right) \\
& -\delta_{n}\left(x_{1} ; p_{2}\right) \geq 0, \quad(i v) \quad \delta_{n}\left(x_{1} ; h_{1}+\varepsilon\right)-\delta_{n}\left(x_{1} ; h_{1}\right) \leq \varepsilon, \quad \text { (v) } \\
& \delta_{n}\left(x_{1} ; h_{0}+\varepsilon\right)-\delta_{n}\left(x_{1} ; h_{0}\right) \geq-T \varepsilon \text {, and (vi) } \delta_{n}\left(x_{1} ; K+\varepsilon\right)-\delta_{n} \\
& \left(x_{1} ; K\right) \geq-\varepsilon .
\end{aligned}
$$

Lemma 5(i), for example, says that $\delta_{n-1}\left(x_{1} ; T\right)$ decreases by at most $\varepsilon\left(\pi+h_{0}\right)$ when $T$ increases by $\varepsilon>0$. The inequality $\delta_{n-1}\left(x_{1} ; T+\varepsilon\right)>(n-(T+\varepsilon)) \pi+h_{1}-(T+$ $\varepsilon) h_{0}-K$ holds for $\varepsilon>0$ if it holds for $\varepsilon=0$. This proves that the hold-back level is nondecreasing in $T$. Similar arguments built on Lemma 5 lead to the rest of Theorem 3.

THEOREM 3. The hold-back level at retailer 1 is nondecreasing in transshipment time $T$, demand probability $p_{1}$, transportation cost $K$, and holding cost $h_{0}$. This level is nonincreasing in holding cost $h_{1}$.

Positive hold-back levels prevent excessive transshipments, for example, when the remaining number of periods until the next replenishment is low. By using hold-back levels and encouraging waiting for the regular replenishment, the IM can reduce transshipment costs. A second reason for having positive hold-back levels is future demands. After sending a transshipment, retailer 1 herself may stock-out and 
Figure 3 Impact of $p_{1}$ on $\tilde{x}_{1}^{n}$ for $p_{2}=0.1, T=4, h_{1}=h_{0}=1, \pi=10$, $K=40$

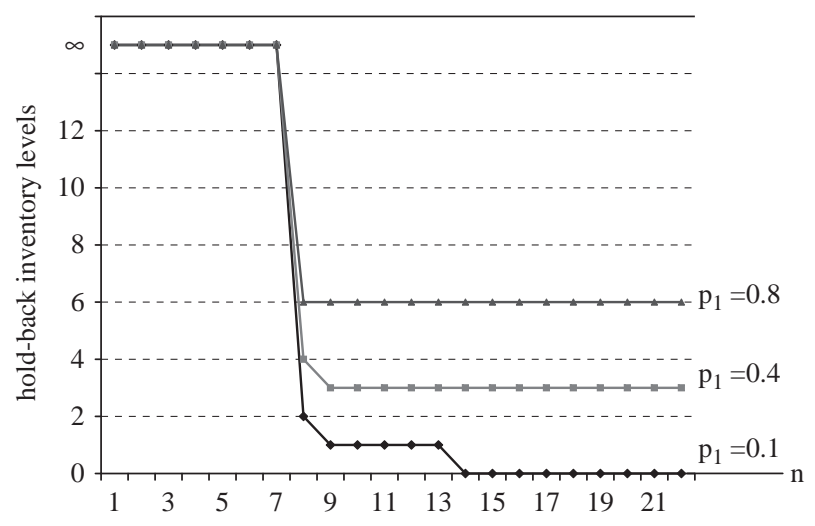

incur a backorder cost. Therefore, it is intuitive that hold-back levels at retailer 1 result more from the consideration of expected demand at retailer 1 than the consideration of demand at retailer 2 . The impact of the demand probability $p_{1}$ on optimal hold-back levels at retailer 1 is illustrated in Figure 3, where $N=22$ and $\tilde{x}_{1}^{n}=\infty$ for $n \leq 7$ as in Theorem 1(ii). Theorem 3 is mute on the sensitivity wrt $p_{2}$, which is further studied starting with Lemma 6.

LEMMA 6. One and only one of the following two statements must hold.

$$
\begin{aligned}
\delta_{n}\left(x_{1} ; p_{2}\right)= & \delta_{n}\left(x_{1} ; p_{2}+\varepsilon\right)>(n+1-T) \pi \\
& +h_{1}-T h_{0}-K, \quad \text { or }
\end{aligned}
$$

$$
\begin{aligned}
\delta_{n}\left(x_{1} ; p_{2}\right) & \leq \delta_{n}\left(x_{1} ; p_{2}+\varepsilon\right) \\
& \leq(n+1-T) \pi+h_{1}-T h_{0}-K,
\end{aligned}
$$

for $\varepsilon>0, n \in\{0, \ldots, N\}, x \in \mathscr{N}$.

Lemma 6 states that if a request is rejected (respectively, accepted) at the current value of $p_{2}$, it is still rejected (respectively, accepted) if $p_{2}$ becomes $p_{2} \pm \varepsilon$. Therefore, the hold-back levels at retailer 1 are robust against changes in demand probability $p_{2}$, which is stated as the next theorem.

THEOREM 4. A retailer's hold-back level is insensitive to the other retailer's demand probability.

For an intuitive explanation of the insensitivity of the hold-back levels, consider the cases of rejection and acceptance. If a request is rejected in the current period, future requests will also be rejected by Theorem 2. This decouples the benefit of inventory of retailer 1 from the demands at retailer 2 . Then this benefit depends only on the demand probability at retailer 1 but not on the demand probability at retailer 2. Nor does marginal cost of rejection depend on the demand probability at retailer 2 , because this cost is
Figure 4 Impact of $\pi$ on $\tilde{x}_{1}^{n}$ for $p_{1}=p_{2}=0.3, T=10, h_{1}=10, h_{0}=0$, $K=10$

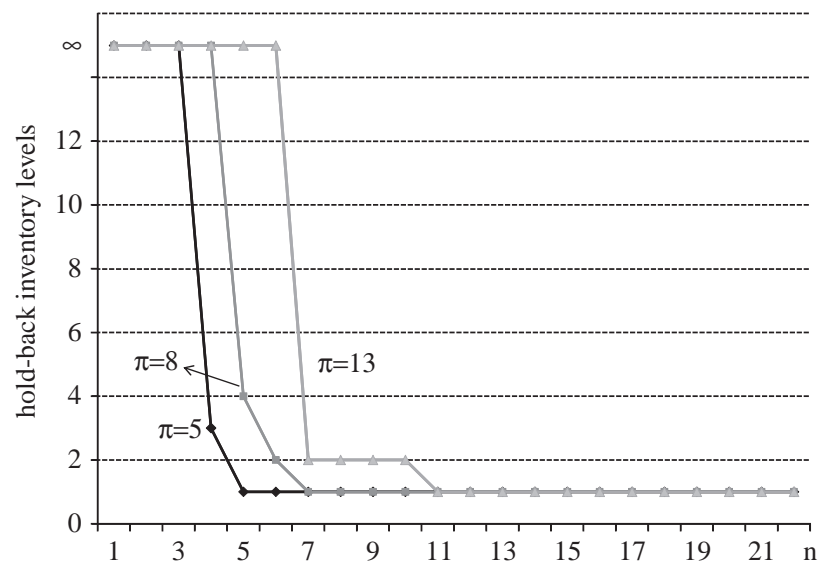

computed given the demand at retailer 2 . Thus, the marginal benefit remains larger than the marginal cost in the case of rejection as the demand probability at retailer 2 increases.

In the case of acceptance, the marginal benefit of that inventory is less than or equal to the marginal cost of rejection. While the marginal cost does not depend on the demand probability at retailer 2, the marginal benefit can increase with this probability. This is because the inventory of retailer 1 is more beneficial if retailer 2 experiences more demand in the acceptance case. However, this increase cannot be too much, given that the marginal benefit is bounded by the monotonicity condition $\delta_{n}\left(x_{1}\right) \leq \pi+\delta_{n-1}\left(x_{1}\right)$ in Lemma 3. This bound allows us to argue that the marginal benefit remains less than or equal to the marginal cost for the acceptance case as the demand probability at retailer 2 increases.

The sensitivity of hold-back levels wrt $\pi$ is more complicated than that wrt to the other parameters. This is because an $\varepsilon$ increase in $\pi$ changes $(n-T) \pi+h_{1}-T h_{0}-K$ proportional to $n$, which itself changes over time. Indeed, hold-back levels may rise wrt to $\pi$ as in Figure 4 or drop as in Figure 5 depending on the values of the other parameters.

\subsection{Performance of Optimal Pooling over Generic Pooling Policies}

In this section, the performance of our partial pooling policy wrt no pooling and complete pooling policies is evaluated. For comparison purposes, we use the optimal cost $V_{N}\left(z_{1}^{*}, z_{2}^{*}\right)$ per cycle. Under a no pooling policy $\left(\tilde{x}_{n}=\infty\right)$, demands at a stocked-out retailer are directly backordered until the next replenishment. Complete pooling $\left(\tilde{x}_{n}=0\right.$ for $n \geq T ; \tilde{x}_{n}=\infty$ for $\left.n<T\right)$ is another extreme policy where a unit is always transshipped to meet the demand at a stocked-out retailer, if the other retailer has on-hand inventory and 
Figure 5 Impact of $\pi$ on $\tilde{x}_{1}^{n}$ for $p_{1}=p_{2}=0.3, T=4, h_{1}=1, h_{0}=1$, $K=40$

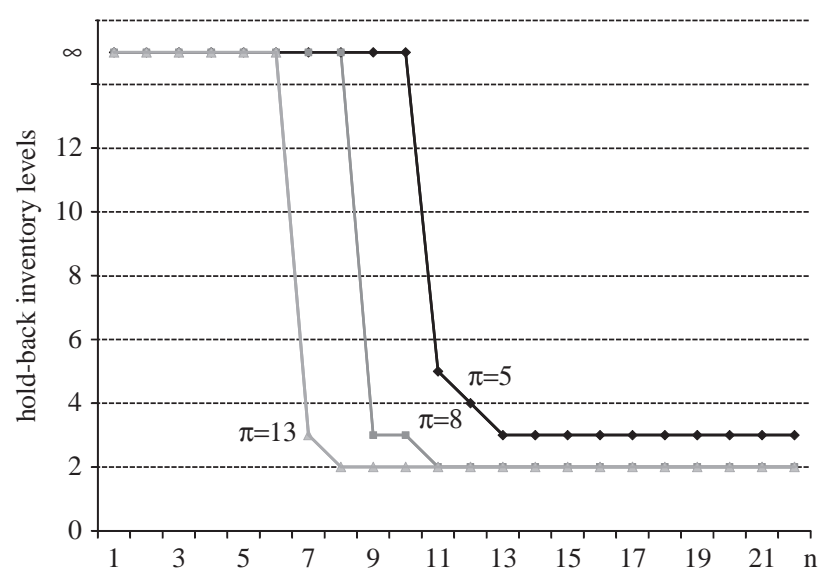

the number of remaining periods is at least $T$. Thus, the complete pooling policy used for comparison in our experimental analysis carries a flavor of our optimal policy as given by Theorem 1(ii). Let $V^{*}=V_{N}\left(z_{1}^{*}, z_{2}^{*}\right)$, and $V_{N P}$ and $V_{C P}$ be the total costs when no pooling and complete pooling policies are used, respectively. Note that for the extreme policies, the costs reported are computed with their respective optimal replenishment quantities. Let $\Delta V_{N P}$ and $\Delta V_{C P}$ denote the improvement in total cycle cost provided by the optimal transshipment policy over no pooling and complete pooling policies, respectively. They are calculated as $\Delta V_{N P}=\left(V_{N P}-V^{*}\right) / V_{N P} \times 100$ and $\Delta V_{C P}=\left(V_{C P}-V^{*}\right) / V_{C P} \times 100$

System costs are evaluated at several different values of each parameter. We report 23 problem instances named P0-P22 in the leftmost column of Table 2, which summarizes the results for the numerical experiments. For each set of parameters, the optimal basestock levels at retailers 1 and 2 are given for optimal, no pooling, and complete pooling.

The transshipment policy does not have a significant impact on basestock levels as shown in the experimental results of Table 2. This is an unexpected observation, but often stated in previous transshipment studies. For example, Grahovac and Chakravarty (2001) mention the surprising result that when transshipments are allowed, retailers experience stable or even increasing inventory levels. Zhao et al. (2005) report that as incentives for inventory sharing increase, retailers respond by decreasing their holdback levels, while they keep their basestock levels constant. Also see Theorem 1 and the ensuing discussion in Zhang (2005). However, as expected, independent from the pooling policy used, the stocking levels tend to increase as (i) demand rates $p_{1}$ and $p_{2}$ increase, (ii) per unit holding costs $h_{1}$ and $h_{2}$ decrease, and (iii) per unit delay cost $\pi$ increases.
The results indicate that cost improvements provided by optimal pooling over no pooling and complete pooling policies mainly have opposite trends. According to the parameter values studied, optimal pooling may perform similar to no pooling (complete pooling), in which case the improvement wrt no pooling (complete pooling) is small and wrt complete pooling (no pooling) is large. For example, optimal pooling results approach no pooling results as $T$ increases in Table 2.

While keeping the total demand rate constant, if the demand symmetry is increased by going from P4 to P7, the improvement wrt complete pooling increases. Complete pooling appears to be more reasonable for asymmetric retailers, while symmetric retailers seem to benefit more from optimal pooling.

The results obtained from the experiments in Table 2 are summarized as follows. (i) As demand rates increase, less transshipment occurs by Theorem 3 and the optimal pooling policy performs similar to the no pooling policy. (ii) As retailers have more symmetric demand rates, optimal pooling provides a better performance wrt the complete pooling policy. (iii) As per unit holding costs $h_{1}$ and $h_{2}$ increase, retailers share more inventory by Theorem 3, so optimal pooling performs similar to the complete pooling policy. (iv) As transshipment time, holding cost rate during transshipment, or transportation cost of transshipment increase, transshipment becomes less profitable by Theorem 3 and the optimal pooling policy acts similar to the no pooling policy.

The improvement by the optimal policy is also measured for 1000 problem instances with randomly chosen parameter values for each problem. Each parameter is randomly generated using a uniform distribution from the ranges given in Table 3. Our results demonstrate average cost improvements of $5.4 \%$ over no pooling and $2 \%$ over complete pooling provided by the optimal pooling policy for the tested problems, where the highest value of improvement exceeds $17 \%$. These results indicate that by using our robust, easily implementable, and optimal transshipment policy, significant improvements can be provided over the two extreme policies of no pooling and complete pooling, which are very popular in practice.

\section{Treatment of Positive Replenishment Time}

A positive replenishment time links the decisions made in consecutive cycles and complicates the problem. Transshipment decisions during the replenishment time $L$ may affect the inventory distribution of the forthcoming cycle, which is independent of the transshipment policy when $L=0$. The inventory 
Table 2 Performance of Optimal Pooling Policy Over Extreme Policies for $N=60$

\begin{tabular}{|c|c|c|c|c|c|c|c|c|c|c|c|c|c|}
\hline & \multirow[b]{2}{*}{$h_{1}$} & \multirow[b]{2}{*}{$h_{2}$} & \multirow[b]{2}{*}{$h_{0}$} & \multirow[b]{2}{*}{$p_{1}$} & \multirow[b]{2}{*}{$p_{2}$} & \multirow[b]{2}{*}{$T$} & \multirow[b]{2}{*}{$K$} & \multirow[b]{2}{*}{$\pi$} & \multirow{2}{*}{$\frac{\text { Optimal pooling }}{z_{1}^{*}, z_{2}^{*}}$} & \multicolumn{2}{|c|}{ No pooling } & \multicolumn{2}{|c|}{ Complete pooling } \\
\hline & & & & & & & & & & $\Delta V_{N P}$ & $z_{1}, z_{2}$ & $\Delta V_{C P}$ & $z_{1}, z_{2}$ \\
\hline P0 & 1 & 1 & 1 & 0.2 & 0.2 & 4 & 40 & 10 & 12,12 & 5.21 & - & 1.51 & - \\
\hline P1 & & & & 0.1 & & & & & 6,12 & 6.92 & 7,12 & 1.13 & - \\
\hline P2 & & & & 0.3 & & & & & 17,12 & 4.74 & - & 1.77 & - \\
\hline P3 & & & & 0.4 & & & & & 23,11 & 4.04 & 23,12 & 1.90 & - \\
\hline P4 & & & & 0.40 & 0.10 & & & & 23,6 & 4.76 & 23,7 & 1.25 & - \\
\hline P5 & & & & 0.35 & 0.15 & & & & 20,9 & 4.70 & - & 1.59 & - \\
\hline P6 & & & & 0.30 & 0.20 & & & & 17,12 & 4.74 & - & 1.77 & - \\
\hline P7 & & & & 0.25 & 0.25 & & & & 14,14 & 4.74 & 15,15 & 1.90 & 14,15 \\
\hline P8 & 0.4 & 0.4 & & & & & & & 13,13 & 3.83 & 14,14 & 2.32 & 13,14 \\
\hline P9 & 0.7 & 0.7 & & & & & & & 12,12 & 4.92 & 13,13 & 1.98 & 12,13 \\
\hline $\mathrm{P} 10$ & 1.3 & 1.3 & & & & & & & 11,11 & 6.20 & - & 1.35 & - \\
\hline $\mathrm{P} 11$ & & & 0.4 & & & & & & 11,12 & 5.42 & 12,12 & 1.42 & 12,12 \\
\hline $\mathrm{P} 12$ & & & 0.7 & & & & & & 11,12 & 5.31 & 12,12 & 1.46 & 12,12 \\
\hline $\mathrm{P} 13$ & & & 1.3 & & & & & & 12,12 & 5.12 & - & 1.57 & - \\
\hline P14 & & & & & & 2 & & & 11,12 & 7.38 & 12,12 & 1.07 & - \\
\hline P15 & & & & & & 6 & & & 12,12 & 3.74 & - & 1.88 & - \\
\hline P16 & & & & & & 8 & & & 12,12 & 2.62 & - & 2.15 & - \\
\hline P17 & & & & & & & 20 & & 11,12 & 7.17 & 12,12 & 0.68 & - \\
\hline P18 & & & & & & & 60 & & 12,12 & 3.85 & - & 2.67 & - \\
\hline P19 & & & & & & & 80 & & 12,12 & 2.81 & - & 4.09 & - \\
\hline P20 & & & & & & & & 8 & 11,11 & 5.40 & 12,12 & 1.94 & - \\
\hline $\mathrm{P} 21$ & & & & & & & & 12 & 12,12 & 6.44 & - & 1.37 & - \\
\hline P22 & & & & & & & & 14 & 12,12 & 6.39 & 13,13 & 1.27 & - \\
\hline
\end{tabular}

$\Delta V_{N P}$ and $\Delta V_{C P}$ are in percentages. Only parameter values that differ from those of P0 are reported for P1-P22 and "_- indicates that replenishment levels are the same as $z_{1}^{*}$ and $z_{2}^{*}$.

positions after order placement for cycle $m$ are $\left(z_{1}^{m}, z_{2}^{m}\right)$. At that time, the inventory levels are $\left(y_{1}^{m}, y_{2}^{m}\right)$. A replenishment time $L$ later, at the beginning of cycle $m$, the inventory levels become $\left(z_{1}^{m}-\hat{\xi}_{1}^{L}\left(y_{1}^{m}, y_{2}^{m} ; \tau\right), z_{2}^{m}-\hat{\xi}_{2}^{L}\left(y_{1}^{m}, y_{2}^{m} ; \tau\right)\right)$. Note that $\hat{\xi}_{i}^{L}\left(y_{1}^{m}\right.$, $\left.y_{2}^{m} ; \tau\right)$ is the sum of the demands met and backordered by retailer $i$ during the last $L$ periods in cycle $m-1$. The inventory levels at the time of order placement and order receipt for cycle $m$ are illustrated in Figure 6. The replenishment amounts are $\left(q_{1}^{m}, q_{2}^{m}\right)$, where $q_{i}^{m}=z_{i}^{m}-y_{i}^{m}$ for $i \in\{1,2\}$.

Table 3 Distributions of Random Parameters for Measuring $\Delta V_{N P}$ and $\Delta V_{C P}$ \begin{tabular}{llllllll}
\hline$h_{1}$ & $\mathrm{U}(0.4,1.4)$ & $h_{2}$ & $\mathrm{U}(0.4,1.4)$ & $h_{0}$ & $\mathrm{U}(0.5,1.4)$ & $T$ & $\mathrm{U}(1,6)$
\end{tabular}

\begin{tabular}{llllllll}
$p_{1}$ & $\mathrm{U}(0.1,0.45)$ & $p_{2}$ & $\mathrm{U}(0.1,0.45)$ & $\pi$ & $\mathrm{U}(5,15)$ & $K$ & $\mathrm{U}(20,60)$ \\
\hline
\end{tabular}

With a positive replenishment time, the expected long-run average cost with initial inventory levels $\left(y_{1}^{1}\right.$, $\left.y_{2}^{1}\right)$ and replenishment policy $Z=\left[\left(z_{1}^{1}, z_{2}^{1}\right),\left(z_{1}^{2}, z_{2}^{2}\right), \ldots\right]$ can be obtained by following similar simplifications that led to (7) and (9). This results in the objective function

$$
\begin{aligned}
\min _{Z} \lim _{M \rightarrow \infty} \frac{1}{M} E & {\left[\sum _ { m = 1 } ^ { M } V _ { N } \left(z_{1}^{m}-\hat{\xi}_{1}^{L}\left(y_{1}^{m}, y_{2}^{m} ; \tau\right), z_{2}^{m}\right.\right.} \\
& \left.\left.-\hat{\xi}_{2}^{L}\left(y_{1}^{m}, y_{2}^{m} ; \tau\right)\right)\right] .
\end{aligned}
$$

The inventory levels at the beginning of a cycle depend on the inventory levels at the order placement time in the previous cycle and the transshipment policy. While analyzing the problem under this 


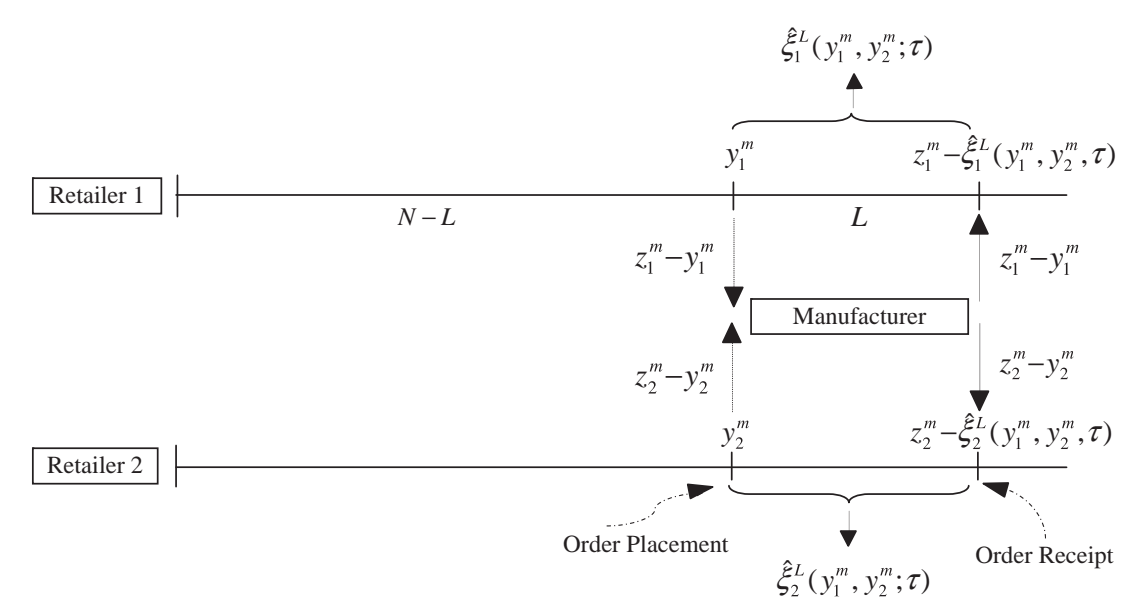

dependence is difficult, results from the problem with $L=0$ can be used for an approximate solution for (13). The IM can solve the transshipment and replenishment problems with zero replenishment lead time by using (10) and obtain the transshipment policy $\tau^{*}$ and the ordering levels $\left(z_{1}^{*}, z_{2}^{*}\right)$. Then the IM can order by also considering the expected demand during replenishment time. The expected demand at retailer $i$ during time $L$ is $p_{i} L$. As a heuristic, the IM can set inventory positions at order placement at $\left(z_{1}^{*}+p_{1} L, z_{2}^{*}+p_{2} L\right)$. The long-run average cost incurred with this heuristic policy is

$$
\begin{aligned}
\lim _{M \rightarrow \infty} \frac{1}{M} E\left\{\sum _ { m = 1 } ^ { M } \left\{V _ { N } \left(z_{1}^{*}+p_{1} L-\hat{\xi}_{1}^{L}\left(y_{1}^{m}, y_{2}^{m} ; \tau^{*}\right),\right.\right.\right. \\
\left.\left.\left.z_{2}^{*}+p_{2} L-\hat{\xi}_{2}^{L}\left(y_{1}^{m}, y_{2}^{m} ; \tau^{*}\right)\right)\right\}\right\} .
\end{aligned}
$$

Since the heuristic policy is feasible, its cost is an UB on (13). It is called the UB heuristic.

To test the UB heuristic, we propose a lower bound by introducing order rebalancing. After the replenishment orders are ready, but just before delivery to the retailers, the order amounts can be reoptimized (i.e., rebalanced), while keeping the total amount delivered to both retailers equal to the total amount ordered. Then the total cost at the retailers is minimized. This reoptimization of orders is called rebalancing, which reduces the effects of unexpectedly lopsided demands during the replenishment time. Rebalancing always reduces cost. More importantly, the dependence of inventory levels at the beginning of a cycle and the transshipment policy can be broken with rebalancing.

When orders are rebalanced, the order amount $z_{i}^{m}-$ $y_{i}^{m}$ is not necessarily delivered to retailer $i$. Let $\beta^{m}$ and $\xi_{1}^{L}+\xi_{2}^{L}-\beta^{m}$ denote the amount of replenishment time demand allocated to retailers 1 and 2, respectively, in cycle $m-1$, where $\xi_{1}^{L}+\xi_{2}^{L}$ is the total random demand during $L$ periods. Consequently, the inventory levels after order receipt at the beginning of cycle $m$ are $\left(z_{1}^{m}-\beta^{m}, z_{2}^{m}-\xi_{1}^{L}-\xi_{2}^{L}+\beta^{m}\right)$. These inventory levels are independent of the transshipment policy $\tau$ or inventory levels $\left(y_{1}^{m}, y_{2}^{m}\right)$. After rebalancing, the manufacturer delivers exactly

$$
\begin{gathered}
q_{1}^{m}=z_{1}^{m}-y_{1}^{m}+\hat{\xi}_{1}^{L}\left(y_{1}^{m}, y_{2}^{m} ; \tau\right)-\beta^{m}, \\
q_{2}^{m}=z_{2}^{m}-y_{2}^{m}+\hat{\xi}_{2}^{L}\left(y_{1}^{m}, y_{2}^{m} ; \tau\right)-\xi_{1}^{L}-\xi_{2}^{L}+\beta^{m} .
\end{gathered}
$$

Note that $q_{1}^{m}+q_{2}^{m}=z_{1}^{m}+z_{2}^{m}-y_{1}^{m}-y_{2}^{m}$. Figure 7 illustrates the reallocated lead time demands, delivered order amounts, and inventory levels at order placement when rebalancing is applied.

The rebalancing concept is used only to find the following lower bound on the average cost:

$\min _{z_{1}, z_{2}} E\left[\min _{\beta}\left\{V_{N}\left(z_{1}-\beta, z_{2}-\xi_{1}^{L}-\xi_{2}^{L}+\beta\right): 0 \leq \beta \leq \xi_{1}^{L}+\xi_{2}^{L}\right\}\right]$.

The following theorem justifies this lower bound, which is valid even without rebalancing.

THEOREM 5. The optimal value of (16) is a lower bound for the average cost in (13).

The convexity of the function in the braces in (16) in $\left(z_{1}, z_{2}\right)$ and $\beta$ follows from the convexity of $V_{n}$. Therefore, optimal replenishment positions and an optimal demand allocation can be obtained from the firstorder optimality conditions.

The gap between the lower bound and the cost of the UB heuristic is computed. The heuristic cost is evaluated for 10,000 cycles by generating random variates to represent the demands over these cycles. Since the demands are integers, inventory positions $\left(z_{1}^{*}+p_{1} L, z_{2}^{*}\right.$ $\left.+p_{2} L\right)$ should be integers. Therefore, the expected lead time demands $\left(p_{1} L, p_{2} L\right)$ are rounded up or down 
Figure 7 Inventory Levels at both Retailers at Order Placement and Order Receipt after Rebalancing

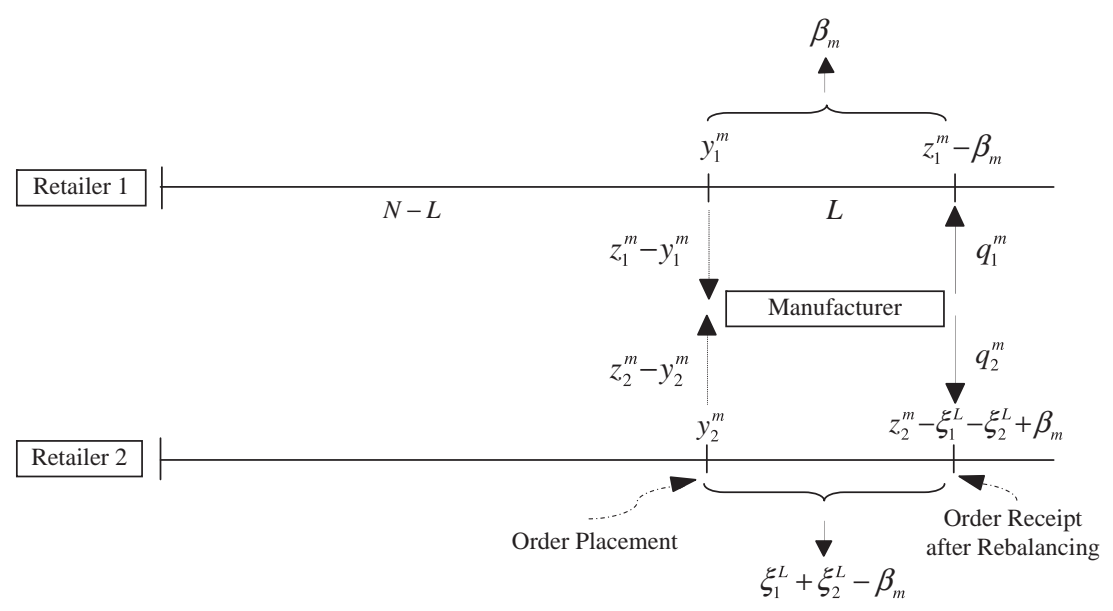

depending on whichever results in a lower longrun average cost. The percent gap is denoted as $\Delta V_{N}^{\text {Bound }}(\%)=\left(V_{N}^{U B}-V_{N}^{L B}\right) / V_{N}^{L B} \times 100$, where $V_{N}^{U B}$ and $V_{N}^{L B}$ are the heuristic cost and lower bound, respectively.

The gap is evaluated for 28 problem scenarios. Twenty-Three were defined in Table 2, all of which have $L=10$. Five additional problems are based on P0 in Table 2. For these problems, $L$ ranges from 6 to 14. The 28 problems and values of $L$ are given in Table 4 . Results indicate that the magnitude of the average gap between the upper and lower bounds is $1.07 \%$. From Table 4, the UB heuristic may on average result in about a $1.07 \%$ increase in total cost over the optimal solution. The gap is smaller for a shorter $L$ and goes to zero as $L$ approaches zero.

The gap between UB and lower bound is also measured with randomly chosen parameter values. Each parameter is generated using a uniform distribution from the ranges given in Table 3 and $L$ is uniformly generated between 6 and 13. For each randomly generated parameter scenario, the lower bound is calculated as the expected cost per cycle and the heuristic cost is evaluated for 5000 cycles. One thousand parameter scenarios are generated randomly and $\Delta$ $V_{N}^{\text {Bound }}(\%)$ is evaluated for each scenario. The results show that the average gap is $1.1 \%$. It is less than $2 \%$ in 95\% of all problem instances and less than $1.5 \%$ in $80 \%$ of all cases. These indicate that in general the UB heuristic cost with positive replenishment time is higher than the optimal cost by a negligible amount.

While the lower bound is obtained by rebalancing inventories after a replenishment, the heuristic replenishment policy does not have a balancing effect. When retailers' basestock levels increase, the gap

Table 4 The Performance of Upper and Lower Bounds for $\boldsymbol{N}=\mathbf{6 0}$

\begin{tabular}{|c|c|c|c|c|c|c|c|c|}
\hline Problem & $L$ & $\Delta V_{N}^{\text {Bound }}(\%)$ & Problem & $L$ & $\Delta V_{N}^{\text {Bound }}(\%)$ & Problem & $L$ & $\Delta V_{N}^{\text {Bound }}(\%)$ \\
\hline P0 & 10 & 0.78 & Increasing $h_{0}$ & & & Increasing $\pi$ & & \\
\hline Increasing $p_{1}$ & & & P11 & 10 & 1.22 & P20 & 10 & 0.95 \\
\hline P1 & 10 & 1.08 & P12 & 10 & 1.34 & P21 & 10 & 0.81 \\
\hline P2 & 10 & 1.08 & P13 & 10 & 1.03 & P22 & 10 & 1.33 \\
\hline P3 & 10 & 1.30 & Increasing $T$ & & & Increasing $L$ & & \\
\hline Decreasing $\left|p_{1}-p_{2}\right|$ & & & P14 & 10 & 0.66 & P0 & 6 & 0.39 \\
\hline P4 & 10 & 0.88 & P15 & 10 & 0.81 & P0 & 8 & 0.57 \\
\hline P5 & 10 & 0.98 & P16 & 10 & 1.18 & P0 & 10 & 0.78 \\
\hline P6 & 10 & 1.08 & Increasing $K$ & & & P0 & 12 & 1.12 \\
\hline P7 & 10 & 1.20 & P17 & 10 & 1.03 & P0 & 14 & 1.47 \\
\hline Increasing $h_{1}, h_{2}$ & & & P18 & 10 & 1.06 & & & \\
\hline P8 & 10 & 2.19 & P19 & 10 & 1.11 & & & \\
\hline P9 & 10 & 1.89 & & & & & & \\
\hline P10 & 10 & 0.69 & & & & & & \\
\hline Average $\Delta V_{N}^{B o u n d}(\%)$ : & & 1.07 & & & & & & \\
\hline
\end{tabular}


between the lower bound and the heuristic may increase as the inventory balancing effect provided by the lower bound may become more observable. As holding costs $h_{1}$ and $h_{2}$ decrease, basestock levels increase as shown in Table 2, the gap becomes larger. As $K$ and $T$ increase, transshipments become less effective in dealing with the inventory imbalance between retailers. As expected, the gap is smaller for a shorter replenishment time $L$ and goes to zero as $L$ approaches zero. In sum, the gap between the lower bound and the cost of the UB heuristic increases when $K, T$, or $L$ increases and $h_{1}$ or $h_{2}$ decreases. These results are consistently observed over 1000 parameter scenarios generated randomly and 28 problem instances reported in Table 4. Although the effect of per period backorder cost $\pi$ on the gap is not monotone in Table 4, according to these 1000 problem instances, as $\pi$ increases, the gap increases in general. Then to observe the combined effect of large $K, T, L, \pi$, and small $h_{1}$ or $h_{2}, 500$ additional problem instances are examined with $K=60, T=6, L=14, \pi=15, h_{1}=h_{2}=0.4$, and randomly generated values of $p_{1}, p_{2}$, and $h_{0}$ by using the ranges given in Table 3 . The gap for these 500 problem instances is $3.11 \%$ on average. This particular value can be interpreted as the worst case performance of the heuristic under the most adverse problem settings tested.

\section{Discussion of Assumptions and Extensions}

Most of our assumptions are made because of practical relevance and analytical tractability. However, they can be relaxed in extensions. Motivated in particular by our personal communications with car makers and dealers of several brands, we consider transshipments only after stock-outs. Transshipment before a stock-out opposes the view of transshipments as an emergency source of supply. In practical contexts where transshipments are viewed as proactive inventory balancing mechanisms and where they are cheap and fast, transshipments before a stock-out can be considered and modeled accordingly.

From the perspective of analysis, each transshipment is assigned to a customer in our current model and a transshipment does not need to be traced after it is initiated. However, if a transshipment were to be initiated before a stock-out, at any time there may be at most $T$ outstanding, unassigned transshipments. Then when making a transshipment decision to satisfy the demand of a current customer, the arrival times for all outstanding transshipments should be considered. To analyze transshipments before a stockout, the record of all outstanding transshipments with their arrival times should be kept, which significantly expands the state space of the problem.
Another extension of our model is to allow simultaneous transshipments of multiple units. In our model, inventories are transshipped in single units. This follows from our context of low-demand products, such as automobiles. Retailers often use third party logistics companies for transshipments. For example, car dealers may use shipping companies such as A-AAA Auto Transport (http://www.autocarmover.com), American Auto Transporters (http://www.shipcar.com), or Auto$\log$ (http://www.autolog.net). Most of these shippers offer very small quantity discounts. "Transporting 3 cars or more at one time entitles ... an additional 5\% discount" according to Autolog. The discount is negligible when compared with the profit made from the sale of a single car. To benefit from such a discount, a dealer can conceivably transship three cars after the third unsatisfied car demand, thus incurring longer waiting times (i.e., additional backorder costs) for the first two demands. However with longer waiting times some of these waiting customers may balk. Another option is to transship three cars after the first unsatisfied demand, where the dealer incurs transshipment costs for two cars whose demands are still uncertain. This latter option corresponds to transshipment before a stock-out. However, there may never be a demand for the extra cars. It is rare in practice and hard to study because of the curse of dimensionality mentioned above.

With a positive transshipment lead time, traceable outstanding transshipments, and sizable quantity discounts for the transportation cost, transshipping multiple units before a stock-out may decrease total costs. It is also practical in some circumstances. Therefore, extending our model to allow transshipment requests at positive inventory levels and transshipping in multiple units is promising.

Another extension is to consider multiple retailers. Then the stocked-out retailer must decide who to request transshipments from. This decision can depend on the inventory levels at the other retailers, so it cannot be made at the beginning of a cycle. In addition, accepting/rejecting a transshipment request is more complicated. If there are multiple retailers with positive inventories, each of them can be more willing to transship wrt the case where there is only one retailer with positive inventory. In other words, the hold-back level of a retailer may be lower because of the opportunity to rely on inventories at other retailers. Such a reliance on inventory levels at other retailers forces the state space to expand. Nevertheless, approximate solutions for the multiple retailer problem can be based on the results in this paper.

\section{Concluding Remarks}

Transshipments are often used in practice to coordinate retailers. Most transshipment research assumes 
Çömez, Stecke, and Çakanyıldırım: Multiple In-Cycle Transshipments with Positive Delivery Times Production and Operations Management 21(2), pp. 378-395, (c) 2011 Production and Operations Management Society

that all transshipments are made at the end of a cycle, after observing all demand in the cycle. Multiple incycle transshipments, which allow a transshipment after each demand realization, are important because in practice, whatever the response to a transshipment request is, a customer would like quick response and would rather not wait until the end of the cycle.

Multiple in-cycle transshipments allow the possibility of using partial pooling policies. Partial pooling policies are studied only recently, perhaps because complete pooling policies are shown (Tagaras and Cohen 1992) to perform relatively better when all transshipments occur at the end of a cycle. We provide numerical studies to illustrate the benefits of our partial pooling policy over two widely used transshipment policies, complete and no pooling.

Our results indicate that when transshipments are considered after each unsatisfied demand, it is optimal for retailers to apply nonstationary transshipment policies defined by hold-back inventory levels. In any period during a cycle, a retailer decides to transship or not to her stocked-out partner according to her on-hand inventory and the hold-back level of that period. The IM computes and communicates this hold-back level by comparing marginal cost and benefit of rejecting a transshipment request. The benefit does not necessarily have a monotone property. Instead, we provide a weaker property, which suffices to obtain the monotonicity of the hold-back levels in time.

We also discuss the complications of a positive replenishment lead time. The interaction between replenishment and transshipment decisions brought by the replenishment lead time is difficult to study. To untangle these decisions, upper and lower bounds on the optimal long-run average cost are developed, where the UB solution is used as a heuristic. The results of an extensive numerical analysis show that the average gap between bounds is small enough to support the use of the UB heuristic solution, which is easy to obtain.

Our results apply to settings with positive transshipment time, positive replenishment lead time, multiple in-cycle transshipments, and periodically incurred backorder and holding costs. The inventory sharing systems developed in this paper for both zero and positive replenishment times are easy to implement. The hold-back levels depend only on the retailers' cost and demand parameters. They are the same over all cycles, independent of the retailers' replenishment quantities or materialized demands in a cycle. Because of this robustness, they do not need to be computed in real time. They can be computed once and kept in a spreadsheet until key parameters change. The IM can compute and communicate them to the retailers once for all cycles.

\section{Acknowledgments}

The authors thank the three anonymous referees, the senior editor, and the editor for their suggestions, which have improved the exposition of the paper. They are also grateful to Paul Zipkin for helpful suggestions.

\section{References}

Archibald, T. W., S. A. E. Sassen, L. C. Thomas. 1997. An optimal policy for a two depot inventory problem with stock transfer. Manage. Sci. 43(2): 173-183.

Arslan, H., S. C. Graves, T. A. Roemer. 2007. A single-product inventory model for multiple demand classes. Manage. Sci. 53(9): 1486-1500.

Berman, O., D. Krass, M. M. Tajbakhsh. 2011. On the benefits of risk pooling in inventory management. Prod. Oper. Manag. 20(1): 57-71.

Bitran, G. R., S. V. Mondschein. 1997. Periodic pricing of seasonal products in retailing. Manage. Sci. 43(1): 64-79.

Chandukala, S. R., J. Kim, T. Otter, P. E. Rossi, G. M. Allenby. 2008. Choice models in marketing: Economic assumptions, challenges, and trends. Found. Trends Mark. 2(2): 97-184.

Çömez, N., M. Çakanyıldırım, K. E. Stecke. 2011a. Unnecessity of transshipments to meet backorders to minimize costs under periodic holding cost accounting. Working Paper \#2009-09, Faculty of Business Administration, Bilkent University, Turkey.

Çömez, N., K. E. Stecke, M. Çakanyıldırım. 2010. Meeting correlated spare part demands with optimal transshipments. Int. J. Strat. Decis. Sci. 1(2): 1-27.

Corsten, D., T. Gruen. 2004. Stock-outs cause walkouts. Harv. Bus. Rev. 82(5): 26-28.

Das, C. 1975. Supply and redistribution rules for two-location inventory systems: One period analysis. Manage. Sci. 21(7): 765776.

Deshpande, V., M. A. Cohen, K. Donohue. 2003. A threshold inventory rationing policy for service-differentiated demand classes. Manage. Sci. 49(6): 683-703.

Duran, A., G. Gutierrez, R. I. Zequeira. 2004. A continuous review inventory model with order expediting. Int. J. Prod. Econ. 87(2): 157-169.

Grahovac, J., A. Chakravarty. 2001. Sharing and lateral transshipment of inventory in a supply chain with expensive lowdemand items. Manage. Sci. 47(4): 579-594.

Gross, D. 1963. Centralized inventory control in multilocation supply systems. Scarf, H. E., D. M. Gilford, M. W. Shelly eds, Multistage Inventory Models and Techniques. Stanford University Press, Stanford, CA, 47-84.

Herer, Y. T., A. Rashit. 1999. Lateral stock transshipments in a twolocation inventory system with fixed and joint replenishment costs. Nav. Res. Logist. 46: 525-547.

Hsu, V. N., C.-L. Li, W.-Q. Xiao. 2005. Dynamic lot size problems with one-way product substitution. IIE Trans. 37: 201-215.

Karmarkar, U. S. 1987. The multilocation multiperiod inventory problem: Bounds and approximations. Manage. Sci. 33: 86-94.

Klein, R. S. 1990. Multilocation Inventory Systems with Transshipments in Response to Stock-out. Unpublished doctoral dissertation, Columbia University, New York, NY.

Krishnan, K. S., V. R. K. Rao. 1965. Inventory control in N warehouses. J. Ind. Eng. 16: 212-215.

Kukreja, A., C. P. Schmidt, D. M. Miller. 2001. Stocking decisions for low-usage items in a multilocation inventory system. Manage. Sci. 47(10): 1371-1383.

Lee, H. L., C. S. Tang. 1998. Variability reduction through operations reversal. Manage. Sci. 44(2): 162-172. 
Lee, T. C., M. Hersh. 1993. A model for dynamic airline seat inventory control with multiple seat bookings. Trans. Sci. 27(3): $252-265$.

Maglaras, C., J. Meissner. 2006. Dynamic pricing strategies for multiproduct revenue management problems. Manuf. Serv. Oper. Manage. 8(2): 136-148.

Minner, S., E. A. Silver. 2005. Evaluation of two simple extreme transshipment strategies. Int. J. Prod. Econ. 93-94: 1-11.

Moinzadeh, K., S. Nahmias. 1988. A continuous review model for an inventory system with two supply modes. Manage. Sci. 34(6): 761-773.

Ozdemir, D., E. Yucesan, Y. T. Herer. 2006. Multi-location transshipment problem with capacitated transportation. Eur. J. Oper. Res. 175: 602-621.

Presman, E., S. P. Sethi. 2006. Inventory models with continuous and Poisson demands and discounted and average costs. Prod. Oper. Manag. 15(2): 279-293.

Rao, U. S., J. M. Swaminathan, J. Zhang. 2004. Multi-product inventory planning with downward substitution, stochastic demand and setup costs. IIE Trans. 36: 59-71.

Righter, R., J. G. Shanthikumar. 2001. Optimal ordering of operations in a manufacturing chain. Oper. Res. Lett. 29: 115-121.

Robinson, L. W. 1990. Optimal and approximate policies in multiperiod, multilocation inventory models with transshipments. Oper. Res. 38(2): 278-295.

Rudi, N., S. Kapur, D. F. Pyke. 2001. A two-location inventory model with transshipment and local decision making. Manage. Sci. 47(12): 1668-1680.

Stalk, G., S. G. Stephenson, T. King. 1997. Searching for fulfillment: Order-to-delivery opportunities in the auto industry. Supply Chain Manage. Rev. September.

Tagaras, G. 1999. Pooling in multilocation periodic inventory distribution systems. Omega, Int. J. Manage. Sci. 27: 39-59.

Tagaras, G., M. A. Cohen. 1992. Pooling in two-location inventory systems with non-negligible replenishment lead times. Manage. Sci. 38(8): 1067-1083.

Tagaras, G., D. Vlachos. 2002. Effectiveness of stock transshipment under various demand distributions and nonnegligible transshipment times. Prod. Oper. Manag. 11(2): 183-198.

Talluri, K., G. van Ryzin. 1998. An analysis of bid-price controls for network revenue management. Manage. Sci. 44(11): 1577-1593.
Toyota.com. 2007. Toyota reports October sales. Available at: http:/ / www.toyota.com/about/news/corporate/2007/11/01-1-sales. html (accessed May 16, 2011).

$\mathrm{Wu}$, P., J. D. Tew. 2005. Collaborations in the automotive after-sales supply chain. Proceedings of the Sixteenth Annual Conference of POMS, Chicago, IL.

Xu, K., P. T. Evers, M. C. Fu. 2003. Estimating customer service in a two-location continuous review inventory model with emergency transshipments. Eur. J. Oper. Res. 145: 569-584.

Zhang, J. 2005. Transshipment and its impact on supply chain members' performance. Manage. Sci. 51(10): 1534-1539.

Zhao, H., V. Desphande, J. K. Ryan. 2005. Inventory sharing and rationing in decentralized dealer networks. Manage. Sci. 51(4): 531-547.

Zhao, H., J. K. Ryan, V. Deshpande. 2006. Emergency transshipments in decentralized dealer networks: When to send and accept transshipment requests. Nav. Res. Logist. 53: 547-567.

Zhao, H., J. K. Ryan, V. Desphande. 2008. Optimal dynamic production and inventory transshipment policies for a two-location make-to-stock system. Oper. Res. 56(2): 400-410.

Zheng, Y.-S. 1994. Optimal control policy for stochastic inventory systems with Markovian discount opportunities. Oper. Res. 42(4): 721-738.

\section{Supporting Information}

Additional supporting information may be found in the online version of this article:

Appendix S1. Estimation and Convergence of the Demand.

\section{Appendix S2. Proofs of Lemmas/Theorems.}

Please note: Wiley-Blackwell is not responsible for the content or functionality of any supporting materials supplied by the authors. Any queries (other than missing material) should be directed to the corresponding author for the article. 